W. Heinzer, C. Rotthaus and J. D. Sally

Nagoya Math. J.

Vol. 131 (1993), 1-38

\title{
FORMAL FIBERS AND BIRATIONAL EXTENSIONS
}

\author{
WILLIAM HEINZER, CHRISTEL ROTTHAUS AND \\ JUDITH D. SALLY ${ }^{1}$
}

\section{Introduction}

Suppose $(R, \mathbf{m})$ is a local Noetherian domain with quotient field $K$ and $\mathbf{m}$-adic completion $\hat{R}$. It is well known that the fibers of the morphism $\operatorname{Spec}(\hat{R}) \rightarrow \operatorname{Spec}(R)$, i.e., the formal fibers of $R$, encode important information about the structure of $R$. Perhaps the most important condition in Grothendieck's definition of $R$ being excellent is that the formal fibers of $R$ be geometrically regular. Indeed, a local Noetherian ring is excellent provided it is universally catenary and has geometrically regular formal fibers [G, (7.8.3), page 214]. But the structure of the formal fibers of $R$ is often difficult to determine. We are interested here in bringing out the interrelatedness of properties of the generic formal fiber of $R$ with the existence of certain local Noetherian domains $C$ birationally dominating $R$ and having $C / \mathrm{m} C$ is a finite $R$-module.

The possible dimensions of the formal fibers of a local Noetherian ring are considered in [Ma2] and in [R3]. Following Matsumura in [Ma2], we use $\alpha(A)$ to denote the maximal dimension of a formal fiber of a local Noetherian ring $A$. If $(R, \mathbf{m})$ is a local Noetherian domain with quotient field $K$ and $\mathbf{m}$-adic completion $\hat{R}$, then Matsumura shows [Ma2, Corollary 1, page 262] that $\alpha(R)$ is the dimension of the generic formal fiber $\hat{R}[K]$. He also observes that if $R$ is of positive dimension, then $\alpha(R) \leq \operatorname{dim}(R)-1$, and he proves that if $R$ is a local ring of positive dimension and finite type over a field, then $\alpha(R)=\operatorname{dim}(R)-1[\mathrm{Ma} 2$, Theorem 2].

If $k$ is a field, $n>r \geq 1$ are integers, and $x_{1}, \ldots, x_{n}$ are indeterminates, then two interesting examples considered in [Ma2] are the rings

$$
A_{r, n}=k\left[\left[x_{1}, \ldots, x_{r}\right]\right]\left[x_{r+1}, \ldots, x_{n}\right]_{\left(x_{1}, \ldots, x_{n}\right)}
$$

Received May 7, 1992.

1 The authors gratefully acknowledge partial support from the National Science Foundation while this work was done. The second author also thanks Purdue University for its hospitality during her visit. 
and

$$
B_{r, n}=k\left[x_{1}, \ldots, x_{r}\right]_{\left(x_{1}, \ldots, x_{r}\right)}\left[\left[x_{r+1}, \ldots, x_{n}\right]\right] .
$$

For each of these $n$-dimensional regular local rings, Matsumura proves that the dimension of the generic formal fiber is $n-2$. He then raises the question of the existence of local Noetherian rings $A$ such that $0<\alpha(A)<\operatorname{dim}(A)-2$. In answer to this question, examples are constructed in [R3] to show that for any integers $n, t$ with $0<t<n-2$, there exists an excellent regular local ring of dimension $n$ for which the generic formal fiber has dimension $t$.

If $\mathbf{p}$ is a prime ideal in the generic formal fiber of $R$, then a natural construct related to $\mathbf{p}$ is the birational extension $C=(\hat{R} / \mathbf{p}) \cap K$. More generally, if $\mathbf{a}$ is an ideal in $\hat{R}$ such that each associated prime of $\mathbf{a}$ is in the generic formal fiber of $R$, then $R$ canonically embeds in $\hat{R} / \mathbf{a}$ and the nonzero elements of $R$ are regular elements of $\hat{R} / \mathbf{a}$, so $K$ canonically embeds in the total quotient ring of $\hat{R} / \mathbf{a}$ and as subrings of the total quotient ring of $\hat{R} / \mathbf{a}$, one can define $C=(\hat{R} / \mathbf{a}) \cap K$. The integral domain $C$ is readily seen to be local, to dominate $R$, and to have as its residue field the field $R / \mathbf{m} \cong \hat{R} / \mathbf{m} \hat{R}$. We are interested in describing $C$ as an extension of $R$. This type of construction has previously been considered in a number of papers [V], [R1], [O1], [O2], [BR], [H], [W]. Using examples of Nagata [Na, Example 5, page 207] and Ogoma [O1], we observe in Section 4 that in general the ring $C=(\hat{R} / \mathbf{p}) \cap K$ need not be Noetherian.

If $\mathbf{a}$ is a finite intersection of prime ideals in the generic formal fiber of $R$, then in addition to $\hat{R} / \mathbf{a}$, it seems natural to also consider the integral closure $B$ of $\hat{R} / \mathbf{a}$ in its total quotient ring. Using a slightly weakened version of Nishimura's criterion for a Krull domain to be Noetherian, we show in Theorems 1.6 and 1.12 that if for all but finitely many of the primes $\mathbf{q} \supset \mathbf{a}$ of $\hat{R}$ the $\operatorname{ring} R /(\mathbf{q} \cap R)$ is complete, then $C=B \cap K$ is a normal analytically unramified semilocal Noetherian domain and $C / \mathbf{m} C$ is a finite-dimensional vector space over $R / \mathbf{m}$.

Taking for $R$ the examples $A_{r, n}$ and $B_{r, n}$ of Matsumura mentioned above, we observe in (1.22) that Theorems 1.6 and 1.12 apply for any ideal $\mathbf{a}$ that is a finite intersection of prime ideals of maximal height in the generic formal fiber and give an uncountable family of 2-dimensional analytically unramified semilocal Noetherian domains birationally containing $R$. In (1.23) and (1.24), we show that Theorems 1.6 and 1.12 apply to certain primes that are maximal in the generic formal fiber of the examples constructed in [R3, Section 3]. This yields, for any integers $n$ and $t$ with $0<t<n-2$, examples $R$ where $\operatorname{dim}(R)=n$, $\mathbf{p}$ is a prime maximal in the generic formal fiber of $R, \mathrm{ht}(\mathbf{p})=t$, and the associated analytically irreducible normal local Noetherian domain $C$ birationally dominating 
$R$ with $C / \mathrm{m} C$ a finite $R$-module is of dimension $n-t$. In fact, we know of no example of an excellent Henselian local domain whose primes maximal in the generic formal fiber fail to satisfy the assumptions of Theorem 1.6. An indication that this might always be true for an excellent Henselian local domain are the results of [HR1] in which we show that an excellent Henselian local domain $R$ with $\alpha(R)=0$ has complete homomorphic images $R / \mathbf{a}$ for any ideal $\mathbf{a}$ of height 2 or larger.

In Theorem 1.26, we present some alternative conditions to those of (1.6) in order that $C=(\hat{R} / \mathbf{a}) \cap K$ be Noetherian. As a corollary, we deduce in (1.27) that if $\operatorname{dim}(\hat{R} / \mathbf{a})=1$, then $C$ is Noetherian and $\hat{C} \cong \hat{R} / \mathbf{a}$. Therefore if $\operatorname{dim}(R)>1$ and $\alpha(R)=\operatorname{dim}(R)-1$, then $R$ is birationally dominated by a one-dimensional local domain $C$ that is analytically ramified, and so, in particular, not a Nagata ring, so not excellent. We observe in Corollary 2.4 that the existence of a dimension one prime ideal $\mathbf{p}$ in the generic formal fiber of $R$ is equivalent to the existence of a rank one discrete valuation domain $V$ that birationally dominates $R$ and has its residue field finite algebraic over $R / \mathbf{m}$.

In Section 2 we make use of the fact that to each semilocal Noetherian domain $C$ birationally containing $R$ with $\mathbf{m}$ contained in the Jacobson radical of $C$ and $C / \mathbf{m} C$ a finite $R$-module, there is naturally associated an ideal $\mathbf{a}$ in $\hat{R}$, the kernel of the canonical map $\phi: \hat{R} \rightarrow \hat{C}$ that extends the inclusion $R \hookrightarrow C$. We observe in (2.2) that $\hat{C}$ is a finite integral extension of $\hat{R} / \mathbf{a}$ inside the total quotient ring of $\hat{R} / \mathbf{a}$. Therefore to each such semilocal Noetherian domain $C$, there is naturally attached an ideal $\mathbf{a}$ in $\hat{R}$ (this ideal $\mathbf{a}$ having the property that each associated prime of $\mathbf{a}$ is in the generic formal fiber of $R$ ) and a finite integral extension $B$ of $\hat{R} / \mathbf{a}$ inside the total quotient ring of $\hat{R} / \mathbf{a}$ such that $C=B \cap K$. In Theorem 2.5, we use these observations together with Theorems 1.6 and 1.12 to deduce for certain local Noetherian domains $(R, \mathbf{m})$ a one-to-one correspondence between prime ideals $\mathbf{p}$ of height $\alpha=\alpha(R)$ in the generic formal fiber of $R$ and analytically irreducible normal local Noetherian domains $C$ such that $C$ birationally dominates $R, \operatorname{dim}(C)=n-\alpha$, and $C / \mathbf{m} C$ is a finite $R$-module. We also obtain a one-to-one correspondence between finite intersections of these prime ideals of height $\alpha$ and normal semilocal analytically unramified Noetherian domains $C$ that birationally dominate $R$ such that each maximal ideal of $C$ has height $\alpha$, and $C / \mathbf{m} C$ is a finite $R$-module. For certain other local Noetherian domains $(R, \mathbf{m})$, we obtain in Theorem 2.7 a one-to-one correspondence between prime ideals $\mathbf{p}$ of $\hat{R}$ that are maximal in the generic formal fiber of $R$ and analytically irreducible normal local Noetherian domains $C$ that birationally dominate $R$ and have $\alpha(C)=0$ and $C / \mathbf{m} C$ is a finite $R$-module. Here again there is an analogous 
statement that applies to ideals that are finite intersections of these primes and to an appropriate family of normal semilocal Noetherian domains which birationally dominate $R$.

In Section 3 we consider conditions under which $C=(\hat{R} / \mathbf{p}) \cap K$ is excellent. In considering geometric regularity of the formal fibers, the field characteristic naturally plays an important role and we have some distinctions according to the characteristic of $R$. We observe in Theorem 3.5 that with $R$ and $\mathbf{p}$ as in Theorem 1.6, if $R$ is excellent and contains a field of characteristic zero, if $\hat{R} / \mathbf{p}$ is normal and each prime ideal $\mathbf{q}>\mathbf{p}$ is extended from $R$, then $C=(\hat{R} / \mathbf{p}) \cap K$ is excellent.

Using examples of Nagata and Ogoma, we show in Section 4 the existence of birational extensions of the form $C=(\hat{R} / \mathbf{p}) \cap K$ which are not Noetherian. From Ogoma's example we obtain two examples, one is the existence of a height 2 prime ideal in the generic formal fiber of a localization of a polynomial ring in 6 variables over a field such that the birational extension associated to this prime is not Noetherian, and the other is a prime ideal of height one in the generic formal fiber of a non-excellent Nagata ring whose associated birational extension is not Noetherian. We observe in $(4.10)$ that if $(R, \mathbf{m})$ is a countable local Noetherian domain, then the generic formal fiber over $R$ is a Jacobson $\operatorname{ring}$, and $\operatorname{dim}(\hat{R} / \mathbf{p})=1$ for each $\mathbf{p} \in \operatorname{Spec}(\hat{R})$ that is maximal in the generic formal fiber of $R$.

Our notation is basically as in [Ma1]. We say that an integral domain $S$ birationally dominates the local domain $(R, \mathbf{m})$ if $R \subseteq S, S$ is contained in the quotient field of $R$, and $\mathbf{m} S$ is contained in the Jacobson radical of $S$.

\section{From generic fiber to birational extension}

Let $(R, \mathbf{m})$ be a local Noetherian domain with quotient field $K$ and suppose $R^{*}$ is a Noetherian extension domain of $R$ that is faithfully flat over $R$. If $\mathbf{a}$ is an ideal of $R^{*}$ such that every associated prime of a lies over 0 in $R$, then $R$ canonically embeds in $R^{*} / \mathbf{a}$ and the nonzero elements of $R$ are regular elements of $R^{*} / \mathbf{a}$. Therefore $K$ canonically embeds in the total quotient ring of $R^{*} / \mathbf{a}$ and we can consider the birational extension $C=K \cap\left(R^{*} / \mathbf{a}\right)$ of $R$.

In terms of elements of $R$ and the ideal $\mathbf{a}$, we note the following.

(1.1). With notation as above and $C=K \cap\left(R^{*} / \mathbf{a}\right)$, we have

$$
C=\left\{a / b: a, b \in R, b \neq 0 \text {, and } a \in \mathbf{a}+b R^{*}\right\} .
$$


Proposition 1.2. Suppose $B$ is an extension ring of $R^{*} / \mathbf{a}$ with the property that every nonzero element of $R$ is a regular element of $B$ and let $C=K \cap B$.

(i) For $a, b \in R, b \neq 0$, we have $a / b \in C \Leftrightarrow a \in b B \Leftrightarrow a$ is in the preimage of $b B$ in $R^{*}$. Therefore a sufficient condition in order that $a / b \in C$ is that $a \in \mathbf{a}+b R^{*}$.

(ii) We have $\left(R^{*} / \mathbf{a}\right)[K]$ is a subring of the total quotient ring of $B$, and if $B^{*}=B \cap\left(R^{*} / \mathbf{a}\right)[K]$, then $B \cap K=B^{*} \cap K$. Therefore in considering the structure of the ring $C=B \cap K$, we may assume that $B \subseteq$ $\left(R^{*} / \mathbf{a}\right)[K]$.

We note the following general facts.

Proposition 1.3. Suppose $B$, as in (1.2), is an extension ring of $R^{*} / \mathbf{a}$ such that every nonzero element of $R$ is a regular element of $B$ and $C=K \cap B$. Then

(i) For any nonzero element $y \in C$, we have $y C=y B \cap C$. In particular, any element of $C$ that is a unit of $B$ is also a unit of $C$, so if $B$ is local, then the contraction to $C$ of the maximal ideal of $B$ is the unique maximal ideal of $C$; and if $B$ is semilocal, then so is $C$ and each maximal ideal of $C$ is the contraction of a maximal ideal of $B$.

(ii) If $S$ is a multiplicatively closed subset of $C$, then $S^{-1} C=K \cap S^{-1} B$. Therefore each prime ideal of $C$ is the union of prime ideals of $C$ that are contracted from $B$. In particular, every prime ideal of $C$ of height one is the contraction of a prime ideal of $B$.

(iii) If $B$ is a Krull domain, then $C=K \cap B$ is a Krull domain, and each height one prime of $C$ is the contraction of a height one prime of $B$. More generally, if $B$ is a finite direct product of Krull domains, then $C$ is a Krull domain and each height one prime of $C$ is the contraction of a height one prime of $B$.

Proof. (i) We note that if $b \in B$ and $y b=x \in C$, then $b=x / y \in K \cap B=C$, so $x \in y C$. The other assertions in (i) follow from this.

(ii) It is clear that $S^{-1} C \subseteq K \cap S^{-1} B$, and if $x \in K \cap S^{-1} B$, then there exists $s \in S$ such that $s x \in K \cap B=C$. Hence $x \in S^{-1} C$.

(iii) It is well known that the intersection of a Krull domain and a subfield of its quotient field is again a Krull domain [B2, page 7], and in the case where $B$ is a Krull domain, the assertion about height one primes follows from well-known properties of the essential valuation rings of a Krull domain [B2, Corollary 2, page 
10]. If $B$ is a finite direct product of Krull domains, say $B=B_{1} \oplus \cdots \oplus B_{n}$, then the fact that every nonzero element of $R$ is regular in $B$ implies that for each $i, i=1, \ldots, n$, the canonical surjection of $B$ to $B_{i}$ restricts to an isomorphism on $R$. Thus $K$ naturally embeds in the quotient field of $B_{i}$ and $C_{i}=K \cap B_{i}$ is well defined. We have $C=\cap_{i=1}^{n} C_{i}$. Therefore $C$ is a Krull domain and each height one prime of $C$ is the contraction of a height one prime of one of the $C_{i}$ and thus also of one of the $B_{i}$.

Theorem 1.4. Suppose $(R, \mathbf{m})$ is a local Noetherian domain, and for $t \in \mathbf{m}$, let $R^{*}=(R, t)^{\wedge}$ denote the completion of $R$ in the topology defined by the powers of the principal ideal tR. Assume that $\mathbf{a}$ is an ideal of $R^{*}$ each of whose associated primes contracts to 0 in $R$, and that $B$ is a finite integral extension of $R^{*} / \mathbf{a}$ having the property that every nonzero element of $R$ is a regular element of $B$. Let $C=K \cap B$. Then $C$ is a semilocal domain and every maximal ideal of $C$ contains $\mathbf{m}$. Let $C^{*}=(C, t)^{\text {^ }}$ denote the completion of $C$ in the topology defined by the powers of $t C$. Then $C^{*}$ is a finitely generated $\left(R^{*} / \mathbf{a}\right)$-submodule of $B$. Therefore $C^{*}$ is Noetherian, $C / t C$ is Noetherian, $C / \mathbf{m} C$ is a finite-dimensional vector space over $R / \mathbf{m}$ and the maximal ideals of $C$ are finitely generated. If $R$ is universally catenary and $\mathbf{a}$ is of positive height, then $C$ is not a spot over $R$.

Proof. Let $x$ be an indeterminate over $B$. We have $R[[x]] \subseteq C[[x]] \subseteq$ $B[[x]]$. Using [Na, (17.5), (16.1)], we have $R^{*}=R[[x]] /(x-t) R[[x]]$ and $C^{*}=$ $C[[x]] /(x-t) C[[x]]$. Since $B$ is a finitely generated $R^{*}$-module, $B$ is complete in the $t B$-adic topology, so that $B \cong B^{*}=B[[x]] /(x-t) B[[x]]$. From part (i) of (1.3) we have that $t B \cap C=t C$. Using this, it follows that

$$
(x-t) B[[x]] \cap C[[x]]=(x-t) C[[x]] .
$$

Therefore the canonical map $C^{*} \rightarrow B^{*}=B$ is an injection. We have

$$
R^{*} \rightarrow C^{*} \hookrightarrow B^{*}=B
$$

with the composite map being finite. Therefore $C^{*}$ is a finitely generated $\left(R^{*} / \mathbf{a}\right)$-submodule of $B$. In particular, $C^{*}$ is Noetherian. Moreover, for any positive integer $n$, we have $t^{n} B \cap C=t^{n} C$ by (1.3)(i), so

$$
R / t^{n} R \rightarrow C / t^{n} C=C^{*} / t^{n} C^{*} \hookrightarrow B / t^{n} B
$$

with the composite map being finite. Therefore $C / t^{n} C$ is a finitely generated $R$-module, so $C / t^{n} C$ is Noetherian and any ideal of $C$ containing a power of $t$ is finitely generated. Since $t \in \mathbf{m}, C / \mathbf{m} C$ is a finitely generated $R / \mathbf{m}$-module, and 
we have the injection

$$
R / \mathbf{m} \hookrightarrow C / \mathbf{m} C=C^{*} / \mathbf{m} C^{*}
$$

Since $B$ is a finite integral extension of $R^{*} / \mathbf{a}, B$ is semilocal, so by (1.3)(i), $C$ is semilocal and every maximal ideal of $C$ is the contraction of a maximal ideal of $B$ and therefore contains $\mathbf{m}$ and thus is finitely generated.

If $R$ is universally catenary, then the dimension formula holds for any spot over $R$ [Ma1, Theorem 15.6, page 119]. Since each maximal ideal of $C$ is the contraction of a maximal ideal of $B$, the residue field with respect to each maximal ideal of $C$ is finite algebraic over $R / \mathbf{m}$; and since $C$ is birational over $R$, if $C$ were a spot over $R$, then $\operatorname{dim}(C)=\operatorname{dim}(R)$. But if $C$ is a spot over $R$, then $C$ is Noetherian and $\operatorname{dim}(C)=\operatorname{dim}\left(C^{*}\right)$. Since $\operatorname{dim}\left(C^{*}\right)=\operatorname{dim}\left(R^{*} / \mathbf{a}\right)$, we conclude that $\mathbf{a}$ of positive height implies $C$ is not a spot over $R$.

Corollary 1.4A. With notation as in Theorem 1.4, if $\operatorname{dim}\left(R^{*} / \mathbf{a}\right)=1$, then $C=B \cap K$ is a one-dimensional semilocal Noetherian domain. Moreover, the completion $\hat{C}$ of $C$ is a subring of $B$ and $B$ is a finitely generated $\hat{C}$-module.

Proof. Since every nonzero element of $R$ is a regular element of $B$, every associated prime of 0 in $B$ contracts to 0 in $R$. From (1.3), it follows that $\operatorname{dim}(C)=1$ and every nonzero prime ideal of $C$ contains $\mathbf{m}$. Therefore $C^{*}=\hat{C}$, so by Theorem $1.4, B$ is a finitely generated $\hat{C}$-module.

We will use the term normal ring as in [Ma1, page 64]. Thus a ring $A$ is normal if for each prime ideal $\mathbf{p}$ of $A$, the localization $A_{\mathbf{p}}$ is an integrally closed domain. It is well known that a normal Noetherian ring is a finite direct product of normal Noetherian domains and thus a finite direct product of Krull domains.

COROLlary 1.4B. With notation as in Theorem 1.4, assume that $B$ is also a normal ring. Then $C=B \cap K$ is a semilocal Krull domain. If $\operatorname{dim}(C)=2$, then $C$ is Noetherian.

Proof. By (1.3)(iii), $C$ is a semilocal Krull domain. By Theorem 1.4, every maximal ideal of $C$ contains $\mathbf{m}$ and is finitely generated. If $\operatorname{dim}(C)=2$, then by Nishimura's criterion [Ni1, Theorem, page 397], $C$ is Noetherian.

We use the following weakened version of Nishimura's criterion [Ni1] for a Krull domain to be Noetherian. 
Lemma 1.5. Let $C$ be a Krull domain. If $C / \mathbf{p}$ is Noetherian for all but at most finitely many of the height one primes $\mathbf{p}$ of $C$, then $C$ is Noetherian.

Proof. It suffices to show that each prime $\mathbf{q}$ in $C$ of height greater than one is finitely generated, for it then follows that $C / \mathbf{p}$ is Noetherian for each height one prime of $C$, and $C$ is Noetherian by [Ni1, Theorem, page 397]. Let $\mathbf{w}_{1}, \ldots, \mathbf{w}_{r}$ be the height one primes of $C$ for which $C / \mathbf{w}_{i}$ may not be Noetherian, and choose $a \in \mathbf{q}-\left(\mathbf{w}_{1} \cup \cdots \cup \mathbf{w}_{r}\right)$. Then $a C=\cap_{j=1}^{s} \mathbf{p}_{j}^{\left(e_{j}\right)}$, where the $\mathbf{p}_{j}$ are height one primes not in the set $\left\{\mathbf{w}_{1}, \ldots, \mathbf{w}_{r}\right\}$. Therefore each $C / \mathbf{p}_{j}$ is Noetherian. By Nishimura [Ni1. Lemma, page 397], it follows that $C / \mathbf{p}_{j}^{\left(e_{j}\right)}$ is Noetherian, and hence by [Na, (3.16)], $C / a C$ is Noetherian. Thus $\mathbf{q}$ is finitely generated.

Question 1. Is it enough to assume in (1.5) that for each prime $\mathbf{q}$ of $C$ there are at most finitely many height one primes $\mathbf{p} \subseteq \mathbf{q}$ such that $C / \mathbf{p}$ is not Noetherian? ${ }^{1}$

We remark that if $A$ is a complete reduced local Noetherian ring, then the integral closure $A^{\prime}$ of $A$ in its total quotient ring is a finite $A$-module. This is clear in view of: (i) the well-known result that this is true in the case where $A$ is an integral domain [Na, (32.1)], (ii) the fact that the total quotient ring $K^{\prime}$ of $A$ is a finite product of fields, say $K^{\prime}=K_{1} \oplus \cdots \oplus K_{n}$, (iii) the fact that the idempotent elements of $K^{\prime}$ are integral over $A$, and (iv) the fact that $A^{\prime}=A_{1}^{\prime} \oplus \cdots \oplus A_{n}^{\prime}$, where $A_{i}$ is the canonical image of $A$ in $K_{i}$ and $A_{i}^{\prime}$ is the integral closure of the complete local domain $A_{\imath}$.

THEOREM 1.6. Suppose $(\boldsymbol{R}, \mathbf{m})$ is a local Noetherian domain with quotient field $K$ and $\mathbf{a}$ is a finite intersection of prime ideals of the completion $\hat{R}$ of $R$ that are in the generic formal fiber of $R$. Suppose $\hat{R} / \mathbf{a} \subseteq B$, where $B$ is a ring normal and finite over $\hat{R} / \mathbf{a}$ and having the property that each minimal prime of $B$ lies over 0 in $R$, or equivalently, having the property that each nonzero element of $R$ is a regular element of $B$. In particular, $B$ can be the integral closure of $\hat{R} / \mathbf{a}$ in the total quotient ring of $\hat{R} / \mathbf{a}$. Let $C=B \cap K$. Assume that for all but at most finitely many of the primes $\mathbf{q} \supseteq \mathbf{a}$ of $\hat{R}$, the ring $R /(\mathbf{q} \cap R)$ is complete. Then we have

(i) $C$ is a normal semilocal Noetherian domain and the residue field at each maximal ideal of $C$ is a finite algebraic extension of $R / \mathbf{m}$. Moreover, any height one prime of $C$ that contains $\mathbf{m} C$ is a maximal ideal of $C$.

1. Added in proof: Ray Heitmann has given an affirmative answer to this question. 
(ii) $\operatorname{dim}(C)=\operatorname{dim}(B)=\operatorname{dim}(\hat{R} / \mathbf{a})$.

(iii) For all but at most finitely many of the height one primes $\mathbf{w}$ of $C$, we have $C / \mathrm{w}$ and $\mathrm{R} /(\mathbf{w} \cap R)$ are complete and $C / \mathbf{w}$ is a finite $\mathrm{R} /(\mathbf{w} \cap R)$ module. Moreover, $C / \mathbf{u}$ is complete for every prime $\mathbf{u}$ of $C$ of height greater than one.

(iv) $C / \mathbf{m} C$ is a finite-dimensional vector space over $R / \mathbf{m}$.

(v) If $R$ is universally catenary (or equivalently quasi-unmixed [Ma1, (31.6) and (31.7]) and if a has positive height, then $C$ is not a spot over $R$.

Proof. Consider the commutative diagram of rings and natural maps:

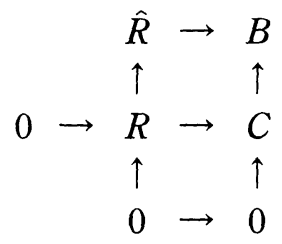

The rows and columns in this diagram are exact, and it follows from (1.2) that $C$ is a semilocal Krull domain and that each height one prime of $C$ is the contraction of a height one prime of $B$. Since $B$ is a finite integral extension of the complete local ring $\hat{R} / \mathbf{a}, B$ is semilocal and the residue field with respect to each maximal ideal of $B$ is finite over $\hat{R} / \mathbf{m} \hat{R} \cong R / \mathbf{m}$. Therefore each maximal ideal of $C$ is the contraction to $C$ of a maximal ideal of $B$ and each residue field with respect to a maximal ideal of $C$ is finite over $R / \mathbf{m}$. Moreover, $\mathbf{m}$ is contained in a height one prime $\mathbf{w}$ of $C$ implies that $\mathbf{w}$ is the contraction of a height one prime of $B$ containing $\mathbf{m}$ and the preimage in $\hat{R}$ of this prime is therefore $\mathbf{m} \hat{R}$. It follows that this height one prime of $B$ is maximal and hence that $\mathbf{w}$ is maximal in $C$.

We note that the preimage in $\hat{R}$ of each height one prime of $B$ is a prime ideal of $\hat{R}$ that properly contains one of the minimal primes of $\mathbf{a}$, and only finitely many height one primes of $B$ have the same preimage in $\hat{R}$. Let $\pi_{1}, \ldots, \pi_{r}$ be the prime ideals of $\hat{R}$ that contain $\mathbf{a}$ and are such that $R /\left(\pi_{i} \cap R\right)$ is not complete, and let $\pi_{1}^{\prime}, \ldots, \pi_{s}^{\prime}$ be the finitely many primes of $B$ which correspond to the $\pi_{i}$. Let $\mathbf{w}$ be a height one prime of $C$ that is not the contraction of one of the $\pi_{i}^{\prime}$. Let $\pi^{\prime}$ be a height one prime of $B$ that contracts to $\mathbf{w}$ in $C$, let $\pi$ be the preimage of $\pi^{\prime}$ in $\hat{R}$, and let $\pi_{0}=\pi \cap R$. Then $R / \pi_{0}$ is complete and $R / \pi_{0}=\hat{R} / \pi_{0} \hat{R} \hookrightarrow \hat{R} / \pi$. Thus $\pi_{0} \hat{R}=\pi$ and we have inclusions $\hat{R} / \pi_{0} \hat{R}=R / \pi_{0} \hookrightarrow C / \mathbf{w} \hookrightarrow B / \pi^{\prime}$. As the composite inclusion $\hat{R} / \pi_{0} \hat{R} \rightarrow B / \pi^{\prime}$ is finite, $C / \mathbf{w}$ is a finite module over $\hat{R} / \pi_{0} \hat{R}=R / \pi_{0}$. Therefore $C / \mathbf{w}$ is complete and Noetherian and $\operatorname{dim}(C / \mathbf{w})=$ $\operatorname{dim}\left(B / \pi^{\prime}\right)$. By Lemma $1.5, C$ is Noetherian. 
Since $B$ is a finite integral extension of $\hat{R} / \mathbf{a}, \operatorname{dim}(B)=\operatorname{dim}(\hat{R} / \mathbf{a})$ and $B$ is a complete semilocal Noetherian ring; and since $B$ is also normal, $B$ is a finite product of complete normal local Noetherian domains (cf. [Na, (17.7)]). We have seen that for all but at most finitely many of the height one primes $\pi$ of $B$, we have $\operatorname{dim}(B / \pi)=\operatorname{dim}(C / \mathbf{w})$, where $\mathbf{w}=\pi \cap C$. Since we have $\operatorname{dim}(B)=1+$ $\max \{\operatorname{dim}(B / \pi)\}$ and $\operatorname{dim}(C)=1+\max \{\operatorname{dim}(C / \mathbf{w})\}$, where $\pi$ and $\mathbf{w}$ range over these sets, we see that $\operatorname{dim}(B)=\operatorname{dim}(C)$. We have also seen that $C / \mathbf{w}$ is complete and a finite module over $R /(\mathbf{w} \cap R)$ for all but at most finitely many of the height one primes $\mathbf{w}$ of $C$. If $\mathbf{u}$ is a prime of $C$ of height greater than one, then $\mathbf{u}$ contains infinitely many height one primes of $C$. It follows that the only primes of $C$ that contain $\mathbf{m} C$ are maximal, for if $\mathbf{u}$ is a prime ideal of height greater than one, then there exists a height one prime $\mathbf{w}$ of $C$ contained in $\mathbf{u}$ such that $C / \mathbf{w}$ is complete and is a finite module over $R /(\mathbf{w} \cap R)$. Since $C / \mathbf{u}$ is a homomorphic image of $C / \mathbf{w}, C / \mathbf{u}$ is also complete.

We have seen that $C$ is a semilocal Noetherian domain for which the residue field at each maximal ideal is a finite-dimensional vector space over $R / \mathbf{m}$ and that the only prime ideals of $C$ that contain $\mathbf{m} C$ are maximal. It follows that $C / \mathbf{m} C$ is a finite-dimensional vector space over $R / \mathbf{m}$.

If $R$ is universally catenary, then the dimension formula holds for any spot over $R$ [Ma1, Theorem 15.6, page 119]. Since the residue field with respect to each maximal ideal of $C$ is finite algebraic over $R / \mathbf{m}$ by part (i), and since $C$ is birational over $R$, if $C$ were a spot over $R$, then $\operatorname{dim}(C)=\operatorname{dim}(R)$. But by part (ii), $\operatorname{dim}(C)=\operatorname{dim}(\hat{R} / \mathbf{a})$, so $\mathbf{a}$ is of positive height implies $\operatorname{dim}(\hat{R} / \mathbf{a})<$ $\operatorname{dim}(\hat{R})=\operatorname{dim}(R)$. Therefore $C$ is not a spot over $R$.

Question 2. Does the hypothesis on $\mathbf{a}$ in Theorem 1.6 imply that each minimal prime $\mathbf{p}_{i}$ of $\mathbf{a}$ is maximal in generic formal fiber of $R$ ?

Question 3. Suppose $(\boldsymbol{R}, \mathbf{m})$ is an excellent local Henselian domain and $\mathbf{p} \subseteq \hat{R}$ is maximal in the generic formal fiber of $R$, does it follow that there are at most finitely many primes $\mathbf{q} \supseteq \mathbf{p}$ of $\hat{R}$ such that $R /(\mathbf{p} \cap R)$ is not complete? As we remark in (1.14), examples of Nagata show this may fail for an $R$ that is not excellent.

Example 1.6A. Let $(R, \mathbf{m})$ be a local Noetherian domain with $\alpha(R)=$ $\operatorname{dim}(R)-1$. Then any ideal $\mathbf{a}$ in $\hat{R}$ that is a finite intersection of prime ideals of 
maximal height in the generic formal fiber of $R$ satisfies trivially the assumptions of Theorem 1.6.

Matsumura has shown in [Ma2, Theorem 2] that if $(R, \mathbf{m})$ is a local domain of positive dimension that is essentially of finite type over a field (i.e., $R$ is a geometric local domain), then $\alpha(R)=\operatorname{dim}(R)-1$. If $n \geq 2$ and $(R, \mathbf{m})$ is an $n$-dimensional geometric local domain over a field $k_{0}$, then in order to find a birationally dominating DVR $C$ such that $C / \mathbf{m} C$ is a finite $R$-module, we can proceed as follows: using for example [Ma3, Theorem 24, page 89], we obtain an extension field $k$ of $k_{0}$ and a "normalization" $R_{0}$ for $R$

$$
R_{0}=k\left[y_{1}, \ldots, y_{n}\right]_{\left(y_{1}, \ldots, y_{n}\right)} \hookrightarrow R
$$

i.e., $R$ is essentially finite over $R_{0}$ and $\operatorname{dim}\left(R_{0}\right)=\operatorname{dim}(R)=n$. If $\phi: \hat{R}_{0} \rightarrow \hat{R}$ is the canonical map on completions extending the inclusion $R_{0} \hookrightarrow R$, then it follows that $\phi$ is injective and finite. In order to find a prime ideal $\mathbf{p}_{0}$ of $\hat{R}_{0}$ with ht $\left(\mathbf{p}_{0}\right)=n-1$ and $\mathbf{p}_{0} \cap R_{0}=(0)$, we pick elements $w_{1}, \ldots, w_{n-1} \in k\left[\left[y_{n}\right]\right]$ which are algebraically independent over $k\left[y_{n}\right]$. An easy calculation shows that the prime ideal $\mathbf{p}_{0}=\left(y_{1}-w_{1} y_{n}, \ldots, y_{n-1}-w_{n-1} y_{n}\right) \hat{R}_{0}$ is in the generic formal fiber of $R_{0}$. Moreover, we have ht $\left(\mathbf{p}_{0}\right)=n-1$ and $\hat{R}_{0} / \mathbf{p}_{0}=B_{0}$ is a DVR. Since $\hat{R}$ is finite over $\hat{R}_{0}$, there is a prime ideal $\mathbf{p}$ of $\hat{R}$ lying over $\mathbf{p}_{0}$. Since $R$ is essentially finite over $R_{0}$ and $\mathbf{p} \cap R_{0}=(0)$, it follows that $\mathbf{p} \cap R=(0)$. We also have $\hat{R} / \mathbf{p}$ is finite over $\hat{R}_{0} / \mathbf{p}_{0}$, so $(\hat{R} / \mathbf{p})$ is a one-dimensional complete local domain and its integral closure $B$ is a DVR that is a finite extension of $B_{0}$. Let $K_{0} \subseteq K$ denote the fields of fractions of $R_{0} \subseteq R$, respectively, and let $C_{0}=B_{0} \cap K_{0}$ and $C=B \cap K$. Then $C_{0} \subseteq C$ are DVRs that birationally dominate $R_{0} \subseteq R$, and the residue field of $C$ is a finite algebraic extension of $k$, so $C / \mathbf{m} C$ is a finite $R$-module.

We note that if $K$ is separable over $K_{0}$, so in particular if $\operatorname{char}(k)=0$, then $C$ is essentially finite over $C_{0}$. Since the cardinality of a transcendence basis of the power series ring over the polynomial ring is not enumerable, for any geometric local domain $R$ of dimension $n \geq 2$, it follows from (2.3) that this construction provides an uncountable set of DVRs birationally dominating $R$ (even if $R$ is enumerable).

In order to show that the completion of $C$ as in (1.6) lies between $\hat{R} / \mathbf{a}$ and $B$, we need some facts about complete local rings.

\section{Lemma 1.7. Let $I$ be an ideal in a Noetherian ring $A$ and}




$$
0 \rightarrow M^{\prime} \rightarrow M \rightarrow M^{\prime \prime} \rightarrow 0
$$

an exact sequence of finitely generated $A$-modules. If $M^{\prime}$ and $M^{\prime \prime}$ are $I$-adically complete, then so is $M$.

Proof. Let $\hat{A}$ denote the $I$-adic completion of $A$. We have the following commutative diagram with exact rows:

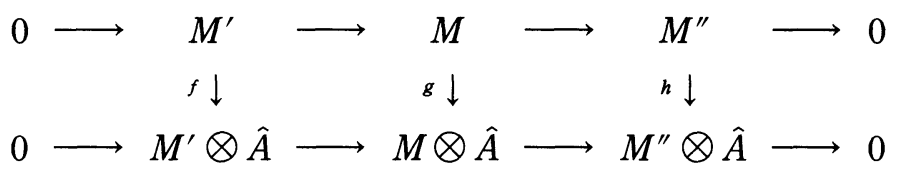

Since $M^{\prime}$ and $M^{\prime \prime}$ are $I$-adically complete, $f$ and $h$ are isomorphisms. By the five lemma [Ma, page 281], $g$ is also an isomorphism, so $M \cong M \otimes \hat{A}$ and $M$ is I-adically complete.

COROLlary 1.8. Let $\mathbf{m}$ be an ideal in a Noetherian ring $A$. If $I, J$ are ideals of $A$ such that $A / I$ and $A / J$ are $\mathbf{m}$-adically complete, then so is $A /(I \cap J)$.

Proof. We have the exact sequence

$$
0 \rightarrow I /(I \cap J) \rightarrow A /(I \cap J) \rightarrow A / I \rightarrow 0
$$

and the isomorphism $I /(I \cap J) \cong(I+J) / J$, so the result follows from (1.7).

Lemma 1.9 ([B1, Exercise 8, page 118]). Let I be an ideal in a Noetherian ring $A$ and let $N$ be the nilradical of $A$. The ring $A$ is I-adically complete if and only if $A / N$ is I-adically complete.

Proof. It is immediate that if $A$ is $I$-adically complete, then so is its homomorphic image $A / N$. To prove the converse, assume that $A / N$ is $I$-adically complete, but $A$ is not. Let $J \subsetneq N$ be maximal with the property that $A / J$ is not $I$-adically complete. Passing from $A$ to $A / J$, we may assume that $J=0$ and that for each nonzero $a \in N, A / a A$ is $I$-adically complete. Choose $a \in N$ such that $a \neq 0$, but $a^{2}=0$, and consider

$$
0 \rightarrow a A \rightarrow A \rightarrow A / a A \rightarrow 0
$$

By assumption, $A / a A$ and $a A$ are $I$-adically complete. It follows from Lemma 1.7 that $A$ is also $I$-adically complete. This contradiction completes the proof. 
Corollary 1.10. Let $\mathbf{m}$ be an ideal in a Noetherian ring $A$. If $A / \mathbf{p}$ is $\mathbf{m}$-adically complete for each minimal prime $\mathbf{p}$ of $A$, then $A$ is $\mathbf{m}$-adically complete.

Proof. Let $N$ be the nilradical of $A$. By Corollary $1.8, A / N$ is $\mathbf{m}$-adically complete. Then by Lemma $1.9, A$ is $\mathbf{m}$-adically complete.

With notation as in Theorem 1.6, we are interested in saying more about the structure of the normal semilocal Noetherian domain $C$. We use the following fact about completions.

Remark 1.11. Suppose $(R, \mathbf{m})$ is a local Noetherian ring, $I$ is an ideal of $R$, $\hat{R}$ is the $\mathbf{m}$-adic completion of $R$, and $R^{*}=(R, I)^{-}$is the $I$-adic completion of $R$. If $R / I$ is complete in the $\mathbf{m}$-adic topology, then $R^{*}=\hat{R}$.

Proof. If $R / I$ is complete, then by Lemma $1.8, R / I^{n}$ is complete for each positive integer $n$. And the fact that $R / I^{n}$ is complete implies that $R / I^{n}=\hat{R} / I^{n} \hat{R}$. By definition, $R^{*}=\lim \left(R / I^{n}\right)$. Therefore $R^{*}=\lim \left(\hat{R} / I^{n} \hat{R}\right)=\hat{R}$.

THEOREM 1.12. With notation and hypotheses as in Theorem 1.6, let $\mathbf{n}$ denote the Jacobson radical of $C$ and let $\hat{C}$ denote the $\mathbf{n}$-adic completion of $C$. Then we have $\hat{R} / \mathbf{a} \subseteq \hat{C} \subseteq B$. Thus $\hat{C}$ is reduced, so $C$ is analytically unramified; and if $B$ is an integral domain, then the $\mathbf{n}$ adic completion of $C$ is an integral domain, i.e., $C$ is analytically irreducible. Moreover, If $B$ is a domain, or is equidimensional, then $C$ is universally catenary.

Proof. We choose $t \in \mathbf{m}$ such that for all primes $\mathbf{q} \supset(t, \mathbf{a}) \hat{R}, R /(\mathbf{q} \cap R)$ is complete. It follows that $t$ is not in any of the finitely many height one primes $\mathbf{w}$ of $C$ such that $C / \mathbf{w}$ is not complete. Then, as in the proof of (1.4), we have that $C^{*}=(C, t)^{-}$, the completion of $C$ with respect to the powers of $t C$ is contained in $(B, t)^{\wedge} \cong B$. By Corollary $1.10, C / t C \cong C^{*} / t C^{*}$ is $\mathbf{n}$-adically complete. Hence by (1.11), $C^{*}=\hat{C}$ is contained in $B$. We have $R \hookrightarrow C \hookrightarrow B$, so the canonical map $\hat{R} \rightarrow \hat{B}=B$ which has kernel a factors through $\hat{C}$. Therefore $\hat{R} / \mathbf{a} \subseteq \hat{C}$ $\subseteq B$. Since $B$ is reduced, $\hat{C}$ is reduced, and if $B$ is a domain, then $\hat{C}$ is a domain. In order that $C$ be universally catenary, it suffices that $\hat{C}$ be equidimensional [Ma1, Theorem 31.6, page 251]. Since $\hat{R} / \mathbf{a} \subseteq \hat{C} \subseteq B$, if $B$ is equidimensional, then $\hat{C}$ is equidimensional, so $C$ is universally catenary. 
(1.13) If $(R, \mathbf{m}), \hat{R}, \mathbf{a}$, and $C$ are as in Theorem 1.6 , then we remark that $\alpha(C)$, the dimension of the generic formal fiber of $C$, is either 0 or 1 . We have $\alpha(C)=0$ if and only if each minimal prime of $\mathbf{a}$ is maximal in the generic formal fiber of $R$. We do not know whether it can happen in this situation that $\alpha(C)>0$, but if it does, then the generic formal fiber of $C$ is a one-dimensional semilocal ring. A related question is whether the generic formal fiber is always a Hilbert (or Jacobson) ring. If this is true, then the hypothesis of Theorem 1.6 implies that $\alpha(C)=0$.

(1.14) Suppose $(R, \mathbf{m})$ is a local domain with completion $\hat{R}$. In connection with Theorems 1.6 and 1.12 , it would be interesting to have conditions on a prime $\mathbf{p} \subseteq \hat{R}$ in the generic fiber over $R$ that imply that for all but finitely many of the primes $\mathbf{q} \supset \mathbf{p}$ of $\hat{R}$, the ring $R /(\mathbf{q} \cap R)$ is complete. We remark that if this condition holds, then there are no containment relations among the finite number of primes $\mathbf{q}$ of $\hat{R}$ that properly contain $\mathbf{p}$ and are such that $R /(\mathbf{q} \cap R)$ is not complete. For if we have $\mathbf{p}<\mathbf{q}_{1}<\mathbf{q}_{2}$ with $R /\left(\mathbf{q}_{2} \cap R\right)$ not complete, then for each of the infinitely many primes $\mathbf{q}$ with $\mathbf{p}<\mathbf{q}<\mathbf{q}_{2}$, it follows that $R /(\mathbf{q} \cap R)$ is not complete since $R /\left(\mathbf{q}_{2} \cap R\right)$ is a homomorphic of $R /(\mathbf{q} \cap R)$.

It seems to us that it may possibly be that if $R$ is excellent and Henselian and $\mathbf{p} \subseteq \hat{R}$ is maximal in the generic fiber over $R$, that it then follows that there are only a finite number of primes $\mathbf{q} \supset \mathbf{p}$ of $\hat{R}$ such that $R /(\mathbf{q} \cap R)$ is not complete (cf. Section 3). Without the hypothesis that $R$ is excellent, examples given by Nagata [Na, (E3.1), page 206] show that one can have $\alpha(R)=0$ with $R$ a regular local such that $\hat{R}$ is purely inseparable over $R$ and there exist infinitely many primes $\mathbf{q}$ of $\hat{R}$ such that $R /(\mathbf{q} \cap R)$ is not complete.

The following result from $[\mathrm{R}],[\mathrm{Ni} 2]$ plays a role in our considerations.

Lemma 1.15 ([R, Lemma 2, page 174], [Ni2 Lemma 1.2, page 156]. Let $A$ be a Noetherian semilocal ring with Jacobson radical $\mathbf{m}$ and let $\hat{A}$ denote the $\mathbf{m}$-adic completion of $A$. Suppose that $I \subseteq \mathbf{m}$ is an ideal of $A$ and that $A$ is I-adically complete. If $J$ is a nonzero ideal of $\hat{A}$ such that for all sufficiently large positive integers $n$, the ideal $J+I^{n} \hat{A}$ is extended from $A$, i.e., $\left(A \cap\left(J+I^{n} \hat{A}\right)\right) \hat{A}=J+I^{n} \hat{A}$, then $J$ is also extended from $A$, i.e., we have $J=(J \cap A) \hat{A}$.

Remarks 1.16. Suppore $(R, \mathbf{m})$ is a local Noetherian domain and $\hat{R}$ is the completion of $R$. In considering prime ideals or finite intersections of prime ideals in the generic formal fiber of $R$ and the corresponding birational normal semilocal extensions $C$ of $R$, it is of interest to know more about the ideals of $\hat{R}$ that are extended from $R$. We note: 
(1) If $\mathbf{q}$ is an ideal in $\hat{R}$ such that $R /(\mathbf{q} \cap R)$ is complete, then $\mathbf{q}=$ $(\mathbf{q} \cap R) \hat{R}$. Moreover, if $\mathbf{a}$ is any ideal of $\hat{R}$ having the same radical as $\mathbf{q}$, then $\mathbf{q} \cap R$ and $\mathbf{a} \cap R$ have the same radical in $R$. By Lemma 1.9, $R /(\mathbf{q} \cap R)$ is complete iff $R /(\mathbf{a} \cap R)$ is complete. Therefore $\mathbf{a}$ is also extended from $R$.

(2) In relation to Lemma 1.15 , it can happen that $R$ is $I$-adically complete and $\mathbf{p}$ is a prime ideal of $\hat{R}$ such that $\mathbf{p}+I \hat{R}$ is extended from $R$, but $\mathbf{p}$ is not extended from $R$. This is illustrated in Example 1.17.

Example 1.17. Let $k$ be a field, let $x, y, z$ be indeterminates over $k$, and let $R=k[x]_{(x)}[[y, z]]$. Consider $\omega=\sum_{i=0}^{\infty} a_{i} x^{i} \in k[[x]]$, with $a_{0} \neq 0$, such that $\omega, x$ are algebraically independent over $k$. We have $\hat{R}=k[[x, y, z]]$ and $\mathbf{p}=(\omega z-y) \hat{R}$ is a prime ideal in $\hat{R}$. Since $\omega$ is invertible in $k[[x]], \mathbf{p}+y \hat{R}=$ $(z, y) \hat{R}$, an ideal that is extended from $R$. We prove that $\mathbf{p} \cap R=0$.

Proof. Suppose $F \in \mathbf{p} \cap R$, and write

$$
F=\sum_{c=0}^{\infty}\left(\sum_{i+j=c} g_{i j}(x) y^{i} z^{j}\right)
$$

where $g_{i j} \in k[x]_{(x)}$. Substituting $y=\omega z$, we have

$$
F(x, \omega z, z)=\sum_{c=0}^{\infty}\left(\sum_{i+j=c} g_{i j}(x) \omega^{i}\right) z^{c} .
$$

Since $\omega, x$ are algebraically independent over $k$,

$$
F(x, \omega z, z)=0 \Longleftrightarrow \sum_{i+j=c} g_{i j}(x) \omega^{i}=0
$$

for all $c \Leftrightarrow$ each $g_{i j}(x)=0 \Leftrightarrow F(x, y, z)=0$. Therefore $\mathbf{p} \cap R=(y-\omega z) \hat{R} \cap$ $R=0$, and $\mathbf{p}$ is not extended from $R$.

Using (1.15), we observe in Proposition 1.18 that there are many examples for which the hypotheses of Theorem 1.6 is satisfied.

Proposition 1.18. Let $(\boldsymbol{R}, \mathbf{m})$ be an $n$-dimensional local Noetherian domain. Suppose $I$ is a nontrivial ideal of $R$ such that $R$ is complete in the $I$-adic topology. If $\mathbf{p}$ is a nonzero prime ideal in the generic formal fiber of $R \operatorname{such}$ that $\operatorname{dim}(\hat{R} / \mathbf{p})=2$, then for all but at most finitely many of the primes $\mathbf{q} \supseteq \mathbf{p}$ of $\hat{R}$, the ring $R /(\mathbf{q} \cap R)$ is complete. 
Proof. We first observe that $\operatorname{dim}(\mathbf{p}+I \hat{R})=1$; for otherwise $\mathbf{p}+I^{k} \hat{R}$ is $\mathbf{m} \hat{R}$ primary for each positive integer $k$ and hence extended from $R$, and this implies, by (1.15), that $\mathbf{p}$ is extended from $R$, which it is not. Let $\mathbf{q} \supset \mathbf{p}$ be any prime ideal of $\hat{R}$ properly containing $\mathbf{p}$ and not containing $\mathbf{p}+I$. Then for each positive integer $k, \mathbf{q}+I^{k}$ is $\mathbf{m} \hat{R}$-primary and hence extended from $R$, so by (1.15), $\mathbf{q}$ is extended from $R$. Therefore $R /(\mathbf{q} \cap R)$ is a one-dimensional local domain which is complete with respect to the nonzero ideal $I+(\mathbf{q} \cap R) /(\mathbf{q} \cap R)$, so $R /(\mathbf{q} \cap R) \cong \hat{R} / \mathbf{q}$.

(1.19) We note that Theorem 1.6 implies that with $(R, \mathbf{m})$ and $\mathbf{p}$ as in (1.18) there exists a 2 -dimensional normal local Noetherian domain $C$ birationally dominating $R$ such that $C / \mathbf{m} C$ is a finite $R$-module. It is often the case that if $(R, \mathbf{m})$ is an $n$-dimensional local Noetherian domain containing a nonzero ideal $I$ such that $R$ is complete in the $I$-adic topology then $\alpha(R)=n-2$, so there exist prime ideals $\mathbf{p}$ in the generic formal fiber of $R$ such that ht $(\mathbf{p})=n-2$. For example, if $k$ is a field, $n>r \geq 1$ are integers, and $x_{1}, \ldots, x_{n}$ are indeterminates, then in [Ma2, Example 3], Matsumura shows that the $n$-dimensional regular local ring

$$
B_{r, n}=k\left[x_{1}, \ldots, x_{r}\right]_{\left(x_{1}, \ldots, x_{r}\right)}\left[\left[x_{r+1}, \ldots, x_{n}\right]\right]
$$

has $\alpha\left(B_{r, n}\right)=n-2$.

(1.20) In [Ma2, Theorem 3], Matsumura considers the situation where $\left(R_{0}, \mathbf{m}_{0}\right)$ and $(R, \mathbf{m})$ are local Noetherian domains such that $R_{0}$ is a subring of $R, \mathbf{m} \cap R_{0}=\mathbf{m}_{0}, R / \mathbf{m}=R_{0} / \mathbf{m}_{0}, R_{0}$ is complete and $\operatorname{dim}(R)>1, \operatorname{dim}\left(R_{0}\right)>0$. Matsumura proves in this situation that $\alpha(R) \leq \operatorname{dim}(R)-2$. For a certain class of these rings, we observe in (1.21) that we have a situation where the hypothesis of (1.6) is satisfied.

Proposition 1.21. Let $(R, \mathbf{m})$ and $\left(R_{0}, \mathbf{m}_{0}\right)$ be local Noetherian domains as in (1.20). If $\mathbf{p} \in \operatorname{Spec}(\hat{R})$ is in the generic formal fiber of $R$ and $\operatorname{dim}(\hat{R} / \mathbf{p})=2$, then for all but at most finitely many of the primes $\mathbf{q} \supseteq \mathbf{p}$ of $\hat{R}$, the ring $\mathrm{R} /(\mathbf{q} \cap R)$ is complete.

Proof. Since $\mathbf{m}_{0}$ is not contained in $\mathbf{p}$, we have $\operatorname{dim}\left(\hat{R} /\left(\mathbf{p}+\mathbf{m}_{0} \hat{R}\right) \leq 1\right.$. Hence $\mathbf{p}+\mathbf{m}_{0}$ is contained in only finitely many primes of $\hat{R}$. Let $\mathbf{q} \supset \mathbf{p}$ be a prime of $\hat{R}$ that properly contains $\mathbf{p}$ and does not contain $\mathbf{m}_{0}$. We have

$$
R_{0} /\left(\mathbf{q} \cap R_{0}\right) \hookrightarrow R /(\mathbf{q} \cap R) \hookrightarrow \hat{R} / \mathbf{q} .
$$


We note that $\left(\mathbf{q}+\mathbf{m}_{0} \hat{R}\right)$ is $\mathbf{m} \hat{R}$-primary, so $\hat{R} /\left(\mathbf{q}+\mathbf{m}_{0} \hat{R}\right)$ is Artinian and hence a finite $R_{0} / \mathbf{m}_{0}$-module. Since $R_{0}$ is complete it follows (cf. [Ma1, Theorem 8.4] that $\hat{R} / \mathbf{q}$ is a finite $R_{0}$-module. Hence $R /(\mathbf{q} \cap R)$ is a finite module over the complete ring $R_{0} /\left(\mathbf{q} \cap R_{0}\right)$ and therefore complete.

(1.22) We remark that if $k$ is a field, $n>r \geq 1$ are integers, and $x_{1}, \ldots, x_{n}$ are indeterminates, then Proposition 1.12 applies to the ring

$$
A_{r, n}=k\left[\left[x_{1}, \ldots, x_{r}\right]\right]\left[x_{r+1}, \ldots, x_{n}\right]_{\left(x_{1}, \ldots, x_{n}\right)}
$$

as it does also to the ring $B_{r, n}$ discussed in (1.19). In [Ma2, Example 2], Matsumura constructs a family of prime ideals $\mathbf{p}$ in the generic formal fiber of $A_{r, n}$ such that $h t(\mathbf{p})=n-2$. By Theorem 1.6 and Proposition 1.21, these prime ideals $\mathbf{p}$ correspond to 2-dimensional normal local Noetherian domains $C$ birationally dominating and residually finite over $A_{r, n}$. It is interesting to note that for both the example $A_{r, n}$ and the example $B_{r, n}$ of (1.19), the cardinality of the prime ideals $\mathbf{p}$ in the generic formal fiber with $h t(\mathbf{p})=n-2$ is uncountable. Therefore it follows from (2.3) below that the cardinality of the 2-dimensional normal local Noetherian domains birationally dominating and residually finite over these rings is uncountable.

(1.23) In [R3, Section 3] for every $n, t \in \mathbf{N}$ with $0<t<n-2$ there is constructed a local excellent domain

$$
A_{n, t}=R_{s}\left[\left[V_{1}, \ldots, V_{t+1}\right]\right]
$$

with $\operatorname{dim}\left(A_{n, t}\right)=n$ and $\alpha\left(A_{n, t}\right)=t$. Here $R_{s}$ is a local excellent domain of dimension $s+1=n-t-1$ with $\alpha\left(R_{s}\right)=0$. (For notational purposes, $s=$ $\operatorname{dim}\left(R_{s}\right)$ - 1.) The ring $R_{s}$ is not complete, but has the property that $R_{s} / \mathbf{a}$ is complete for each nonzero ideal a of $R_{s}$. The completion $\hat{R}_{s}$ of $R_{s}$ is the formal power series ring $K\left[\left[X_{1}, \ldots, X_{s}, T\right]\right]$, and the completion of $A_{n, t}$ is

$$
\hat{A}_{n, t}=K\left[\left[X_{1}, \ldots, X_{s}, V_{1}, \ldots, V_{t+1}, T\right]\right]
$$

a formal power series ring in $n$ variables over a field $K$ of characteristic 0 .

Using the Weierstrass preparation theorem, as in the proof Claim (a) of [R3, Section 3], it is readily seen that

(*) Any prime ideal $\mathbf{p}$ in $\hat{A}_{n, t}$ that is not contained in $\left(V_{1}, \ldots, V_{t+1}\right) \hat{A}_{n, t}$ is such that $\mathbf{p}$ is extended from $A_{n, t}$ and $A_{n, t} /\left(\mathbf{p} \cap A_{n, t}\right)$ is complete. On the other hand, if $\mathbf{q}=\left(V_{1}, \ldots, V_{t+1}\right) \hat{A}_{n, t}$, then $A_{n, t} /\left(\mathbf{q} \cap A_{n, t}\right)$ has $R_{s}$ as a homomorphic image and hence is not complete. 
It follows that the prime ideals in the generic formal fiber of $A_{n, t}$ are contained in $\left(V_{1}, \ldots, V_{t+1}\right) \hat{A}_{n, t}$ and that the prime ideals $\mathbf{p}$ of maximal height $t$ in the generic formal fiber satisfy the hypothesis of Theorem 1.6. If $\mathbf{p}$ is one of these prime ideals and $B$ is the integral closure of $\hat{A}_{n, t} / \mathbf{p}$ and $L$ is the quotient field of $A_{n, t}$, then by Theorems 1.6 and $1.12, C=B \cap L$ is a normal local Noetherian domain of dimension $n-t=s+2$ and $\hat{A}_{n, t} \subseteq \hat{C} \subseteq B$.

We do not know whether there are maximal ideals of height less than $t$ in the generic formal fiber of $A_{n, t}$. If such a prime ideal $\mathbf{p}$ exists, then for each of the infinitely many primes $\mathbf{q}$ such that $\mathbf{p} \subset \mathbf{q} \subset\left(V_{1}, \ldots, V_{t+1}\right) \hat{A}_{n, t}$, the ring $A_{n, t} /(\mathbf{q} \cap$ $\left.A_{n, t}\right)$ is not complete, so the hypothesis of Theorem 1.6 would not be satisfied for this $\mathbf{p}$.

(1.24) Further applications. In relation to the hypothesis of Theorem 1.6, we consider the generic formal fiber of the ring $B$ of Corollary 3.2 of [R3]. This ring $B$ is an example of an excellent local domain whose generic formal fiber admits maximal ideals of different heights. Building on the ring $A_{n, t}$ of [R3, Section 3] discussed above in (1.23), the ring $B$ is constructed as follows: let $S=A_{n, t}$, let $\mathbf{m}_{S}$ denote the maximal ideal of $S$, let $W$ be a variable, and let $B=S[W]_{\left(\mathbf{m}_{S}, W\right.}$. Recall that we have

$$
\hat{R}_{s}=K\left[\left[X_{1}, \ldots, X_{s}, T\right]\right] \text { and } \hat{S}=\left[\left[X_{1}, \ldots, X_{s}, T, V_{1}, \ldots, V_{t+1}\right]\right] .
$$

We obviously have

$$
\hat{B}=K\left[\left[X_{1}, \ldots, X_{s}, T, V_{1}, \ldots, V_{t+1}, W\right]\right]=\hat{S}[[W]]
$$

and $n=s+t+2$ and $\operatorname{dim}(B)=n+1$. We consider the canonical injections

$$
B=S[W]_{\left(\mathbf{m}_{S}, W\right)} \hookrightarrow \hat{S}[W]_{\left(\mathbf{m}_{\hat{S}}, W\right)} \hookrightarrow \hat{S}[[W]]=\hat{B} .
$$

It follows from [Ma2, Example 2] that the dimension of the generic formal fiber of $\hat{S}[W]_{\left(\mathbf{m}_{\hat{s}}, W\right)}$, is $n-1=\operatorname{dim}(\hat{B})-2$, so $\alpha(B)=n-1=\operatorname{dim}(B)-2$. Using Proposition 1.21, we see that every prime ideal $\mathbf{p}$ of maximal height in the generic formal fiber of $B$ satisfies the hypothesis of Theorem 1.6. Therefore to each of these prime ideals $\mathbf{p}$ of $\hat{B}$ is associated a 2 -dimensional normal local Noetherian domain birationally dominating and residually finite over $B$.

It is shown in [R3, Corollary 3.2] that there exist prime ideals p maximal in the generic formal fiber of $B$ with $h t(\mathbf{p}) \leq t+1$. To see this, take $\mathbf{p}_{0} \in$ $\operatorname{Spec}(\hat{S})$ with $\mathrm{ht}\left(\mathbf{p}_{0}\right)=t$ and $\mathbf{p}_{0}$ in the generic formal fiber of $S$, and $\beta \in K[[T]]$ to be a non-invertible element that is algebraically independent over $S$. Then $\mathbf{p}_{\beta}=\left(W-\beta, \mathbf{p}_{0}\right) \hat{B}$ is a prime ideal of $\hat{B}$ of height $t+1$ and the fact 
that $\beta$ is algebraically independent over $S$ implies that if $L$ is the quotient field of $S$ and $C=\left(\hat{S} / \mathbf{p}_{0}\right) \cap L$, then $\beta$ is algebraically independent over $C$, so $\mathbf{p}_{\beta} \cap$ $B=(0)$ and $\mathbf{p}_{\beta}$ is in the generic formal fiber of $B$.

Now we observe that any prime ideal $\mathbf{p}$ of height $t+1$ which is maximal in the generic formal fiber of $B$ and contains a monic polynomial in $W$ (as, for example, the $\mathbf{p}_{\beta}$ ), then $\mathbf{p}$ satisfies the hypothesis of Theorem 1.6, i.e., for all but at most finitely many prime ideals $\mathbf{q} \supset \mathbf{p}$ of $\hat{B}, B /(\mathbf{q} \cap B)$ is complete. For if $\mathbf{q} \supset \mathbf{p}$, then since $\mathbf{p}$ contains a monic polynomial $f(W)$ in $\hat{S}[W]$, we have $\mathbf{q}$ is a minimal prime of $\left(f(W), \mathbf{q}_{0}\right) \hat{B}$, where $\mathbf{q}_{0}=\mathbf{q} \cap \hat{S}$. If $\mathbf{q}_{0}$ is not contained in $\left(V_{1}, \ldots, V_{t+1}\right) \hat{S}$, then as noted in $(*)$ in $(1.23)$, it follows that $S /\left(\mathbf{q}_{0} \cap S\right)$ is complete, so $S /\left(\mathbf{q}_{0} \cap S\right)=\hat{S} / \mathbf{q}_{0}$. It follows that $B /(\mathbf{q} \cap B)$ is a finite integral extension of $S /\left(\mathbf{q}_{0} \cap S\right)=\hat{S} / \mathbf{q}_{0}$ and hence is also complete. Therefore the only possible prime ideals $\mathbf{q} \supset \mathbf{p}$ of $\hat{B}$ that are such that $B /(\mathbf{q} \cap B)$ is not complete have the property that $\mathbf{q} \cap \hat{S}=\mathbf{q}_{0} \subseteq\left(V_{1}, \ldots, V_{t+1}\right) \hat{S}$. With ht $(\mathbf{p})=t+1$ and $\mathbf{p}$ containing a monic polynomial in $W$, each of these primes $\mathbf{q}$ that properly contains $\mathbf{p}$ is such that $\left(V_{1}, \ldots, V_{t+1}\right) \subset \mathbf{q}$, and there are only finitely many such primes $\mathbf{q}$.

(1.25) We remark that there are two kinds of prime ideals $\mathbf{p}_{i}$ in $B$ that are maximal with the property that $B / \mathbf{p}_{i}$ is not complete. One of these sets consists of just one element, the prime ideal $\mathbf{p}_{1}=\left(X_{1}, \ldots, X_{s}, T, V_{1}, \ldots, V_{t+1}\right) B$, a prime ideal of height $n$ and dimension 1 . The other prime ideals $\mathbf{p}$ of $B$ maximal with respect to the property that $B / \mathbf{p}$ is not complete are all of the form $\mathbf{p}_{2}=\left(V_{1}, \ldots, V_{t+1}, f(W)\right) B$, where $f(W)$ is a monic polynomial of positive degree in $S[W]$. Each of the prime ideals $\mathbf{p}_{2}$ is of height $t+2$ and dimension $s+1$. We ask if there is a connection between the ideals maximal in the generic formal fiber of an excellent local domain $R$ and the set of prime ideals $\mathbf{p} \in \operatorname{Spec}(R)$ for which $R / \mathbf{p}$ is complete. We refer to [HR1] for more investigation into this question.

In Theorem 1.26, we present some alternative conditions to those of (1.6) in order that $C$ be Noetherian.

THEOREM 1.26. Suppose $(R, \mathbf{m})$ is a local Noetherian domain with quotient field $K$ and $\mathbf{m}$-adic completion $\hat{R}$ and $\mathbf{a}$ is an ideal of $\hat{R}$ with the property that each associated prime of $\mathbf{a}$ is in the generic formal fiber of $R$. Suppose $B$ is a finite integral extension of $\hat{R} / \mathbf{a}$ having the property that every nonzero element of $R$ is a regular element of $B$, and let $C=B \cap K$.

(i) If for each nonzero prime ideal $\mathbf{u}$ of $C$ there exists $a \in \mathbf{u} \cap R$ such that $\mathbf{a}+a^{n} \hat{R}$ is extended from $R$ for all sufficiently large positive integers $n$, then $C$ is Notherian. 
(ii) If $B$ is a normal ring and if for each prime ideal $\mathbf{u}$ of $C$ of height greater than one there exists $a \in \mathbf{u} \cap R$ such that $\mathbf{a}+a^{n} \hat{R}$ is extended from $R$ for all sufficiently large positive integers $n$, then $C$ is a normal Noetherian domain.

In both (i) and (ii) we have that the completion $\hat{C}$ of $C$ is such that $\hat{R} / \mathbf{a} \subseteq \hat{C} \subseteq B$.

Proof. Let $\mathbf{u}$ be a prime ideal in $C$ and assume $a \in \mathbf{u} \cap R$ is such that $\mathbf{a}+$ $a^{n} \hat{R}$ is extended from $R$ for all sufficiently large $n$. By (1.15), a is extended from $R^{*}=(R, a)^{-}$, the completion of $R$ with respect to the powers of $a R$. Let $\mathbf{a}^{*}=$ a $\cap R^{*}$. Then $\hat{R} / \mathbf{a}$ is the completion of $R^{*} / \mathbf{a}^{*}$. We note by replacing $B$ if necessary by $B \cap(\hat{R} / \mathbf{a})[K]$, we may assume that $B \subseteq(\hat{R} / \mathbf{a})[K]$. Let $K^{*}$ denote the total quotient ring of $R^{*} / \mathbf{a}^{*}$ and let $B^{*}=B \cap K^{*}$. Since $B$ is a finitely generated $\hat{R} / \mathbf{a}$-module and since $B \subseteq(\hat{R} / \mathbf{a})[K]$, there exists a nonzero element $t \in R$ such that $t B \subseteq \hat{R} / \mathbf{a}$. It follows that $t B^{*} \subseteq R^{*} / \mathbf{a}^{*}$. Therefore $B^{*}$ is a finitely generated $R^{*} / \mathbf{a}^{*}$-module. Since $C=B^{*} \cap K$, by (1.3)(i), we have $a B^{*} \cap C=$ $a C$. Thus

$$
R / a R \rightarrow C / a C \hookrightarrow B^{*} / a B^{*}
$$

with the composite map being finite. Therefore $C / a C$ is a finitely generated $R$-module and hence is Noetherian. It follows that $\mathbf{u}$ is a finitely generated prime ideal of $C$. In (i), we conclude that each prime ideal of $C$ is finitely generated, so $C$ is Noetherian; while in (ii), each prime ideal of $C$ of height $>1$ is finitely generated. Since in (ii), $B$ is a normal ring, $C=B \cap K$ is a Krull domain by (1.3)(iii), and so is Noetherian by [Ni1].

As in Theorem 1.4, we have inclusions of the formal power series rings $R[[x]] \subseteq C[[x]] \subseteq B^{*}[[x]]$ and $R^{*}=(R, a)^{\wedge}=R[[x]] /(x-a) R[[x]], C^{*}=$ $C[[x]] /(x-a) C[[x]]$, and $B^{*} \cong B^{*}[[x]] /(x-a) B^{*}[[x]]$. From part (i) of (1.3), we have $a B^{*} \cap C=a C$, and from this it follows that

$$
(x-a) C[[x]]=(x-a) B^{*}[[x]] \cap C[[x]] .
$$

Therefore the canonical map $C^{*} \rightarrow B^{*}$ is an injection. We have

$$
R^{*} \rightarrow C^{*} \hookrightarrow B^{*}
$$

with $\mathbf{a}^{*}$ the kernel of the map $R^{*} \rightarrow B^{*}$. Thus $R^{*} / \mathbf{a}^{*} \subseteq C^{*} \subseteq B^{*}$. Since $C$ and $C^{*}$ have the same completion $\hat{C}$, we conclude that $\hat{R} / \mathbf{a} \subseteq \hat{C} \subseteq B$.

COROllary 1.27. Suppose $(R, \mathbf{m})$ is a local Noetherian domain with quotient 
field $K$ and $\mathbf{m}$-adic completion $\hat{R}$ and $\mathbf{a}$ is an ideal of $\hat{R}$ with the property that each associated prime of $\mathbf{a}$ is in the generic formal fiber of $R$. Suppose $B$ is a finite integral extension of $\hat{R} / \mathbf{a}$ having the property that every nonzero element of $R$ is a regular element of $B$. If $\operatorname{dim}(\hat{R} / \mathbf{a})=1$, then $C=B \cap K$ is Noetherian, $\operatorname{dim}(C)=1$, and if $\hat{C}$ is the completion of $C$, then $\hat{R} / \mathbf{a} \subseteq \hat{C} \subseteq B$. Therefore, in the case where $B=\hat{R} / \mathbf{a}$, we have $\hat{C} \cong \hat{R} / \mathbf{a}$ and

(i) if $\mathbf{a}$ is prime, then $C$ is analytically irreducible,

(ii) if $\mathbf{a}$ is an intersection of primes, then $C$ is analytically unramified, while

(iii) if $\mathbf{a}$ is properly contained in $\operatorname{rad}(\mathbf{a})$, then $C$ is analytically ramified.

If $(R, \mathbf{m})$ is any local Noetherian domain of dimension $n>1$ such that $\alpha(R)=n-$ 1 , then there exists a one-dimensional local Noetherian domain $C$ that birationally dominates $R$ and is analytically ramified.

Proof. Since $\operatorname{dim}(\hat{R} / \mathbf{a})=1$ and each associated prime of $\mathbf{a}$ is in the generic formal fiber of $R$, the only prime ideal of $\hat{R}$ containing a that has a nonzero contraction to $R$ is the maximal ideal $\mathbf{m} \hat{R}$. From (1.3)(ii) it follows that each height one prime of $C$ is the contraction of a height one prime of $B$. Since $B$ is semilocal and $\operatorname{dim}(B)=1$, by $(1.3) \operatorname{dim}(C)=1$ and each nonzero prime ideal $\mathbf{u}$ of $C$ contains $\mathbf{m}$. Since every primary ideal for the maximal ideal of $\hat{R}$ is extended from $R$, it follows that for any nonzero $a \in \mathbf{m}$ the ideal $\mathbf{a}+a \hat{R}$ is extended from $R$. Hence by Theorem 1.26, $C$ is Noetherian and $\hat{R} / \mathbf{a} \subseteq \hat{C} \subseteq B$.

To see the existence of $C=(\hat{R} / \mathbf{a}) \cap K$ that is analytically ramified, we take $\mathbf{p}$ to be any dimension one prime in the generic formal fiber of $R$ and take $\mathbf{a}$ to be any $\mathbf{p}$-primary ideal with $\mathbf{a}<\mathbf{p}$.

Remark 1.28. An argument like the one in Theorem 1.26 is used to show that numerous counterexamples are Noetherian [BR], [H], [O1], [O2], [O3], [R1], [W].

These constructions start with the localization $R=k\left[x_{1}, \ldots, x_{n}\right]_{\left(x_{1}, \ldots, x_{n}\right)}$ of a polynomial ring over an infinite countable field $k$. Following [BR], after choosing a suitable enumeration $\left(p_{i}\right)_{i \in n}$ of a set of representatives of the prime elements in $R$, we set:

$$
q_{n}=\prod_{i=1}^{\mathrm{n}} p_{i} \quad \text { and } \quad \tilde{R}=\bigcap_{i \in \mathbf{N}}\left(R, q_{i}\right)^{-}
$$

Then ideals $\tilde{\mathbf{a}}$ in $\tilde{R}$ are constructed with $\tilde{\mathbf{a}} \cap R=(0)$. The ideal $\tilde{\mathbf{a}} \hat{R}$ satisfies the hypothesis of Theorem 1.26(i), so if $K$ is the quotient field of $R$, then $C=$ 
$(\hat{R} / \tilde{\mathbf{a}} \hat{R}) \cap K$ is a local Noetherian domain. It is also true that $\hat{C}=\hat{R} / \tilde{\mathbf{a}} \hat{R}$. It is shown in $[\mathrm{BR}]$ that there is considerable flexibility in this construction of $\mathbf{a}$. For example, with $R=k[x, y, z, u, w]_{(x, y, z, u, w)}$, it is possible to obtain $\tilde{R}$ and prime ideals $\tilde{\mathbf{a}}$ that extend to $\hat{R}$ to give a chain of prime ideals $\mathbf{p}_{1}<\mathbf{p}_{2}<\mathbf{p}_{3}$ such that ht $\left(\mathbf{p}_{i}\right)=i$ and $\mathbf{p}_{3} \cap R=(0)$. With $C_{i}=\left(\hat{R} / \mathbf{p}_{i}\right) \cap K$, it follows that $C_{1}<C_{2}$ $<C_{3}$ is a chain of normal local Noetherian domains birationally dominating $R$ and having residue field $k$ such that $\operatorname{dim}\left(C_{i}\right)=5-i, 1 \leq i \leq 3$.

To make this construction work the field $k$ has to be countable and infinite and the prime ideals $\mathbf{p}_{i}$ in the generic formal fiber of $R$ have to be chosen careful$1 y$. We show in Section 4 that in general if $\mathbf{p}$ is a prime ideal in the generic formal fiber of $R$ that is not maximal in the generic formal fiber, then $C=(\hat{R} / \mathbf{p}) \cap K$ need not be Noetherian.

\section{From birational extension to generic formal fiber}

In the discussion below relating birational extensions of $R$ to extensions of $\hat{R}$, we use the following remark concerning tensor products.

(2.1) Suppose $(R, \mathbf{m})$ is a local Noetherian domain with quotient field $K$ and $R^{*}$ is an $R$-algebra that is faithfully flat over $R$. Then $R^{*} \bigotimes_{R} K \cong R^{*}[K]$ is a localization of $R^{*}$ inside the total quotient ring $K^{*}$ of $R^{*}$, and for any birational extension $C$ of $R$, we have $R^{*} \otimes_{R} C \cong R^{*}[C]$, the composite of $R^{*}$ and $C$ in $K^{*}$.

(2.2) Suppose $(R, \mathbf{m})$ is a local Noetherian domain with quotient field $K$ and completion $\hat{R}$. If $C$ is a semilocal Noetherian domain birationally dominating $R$ such that $\mathbf{m}$ is contained in the Jacobson radical of $C$ and $C / \mathbf{m} C$ is a finite $R$-module, then there are ideals of $\hat{R}$ and of the birational extension $\hat{R}[C]$ of $\hat{R}$ that are naturally related with $C$. Let $\hat{C}$ denote the $\mathbf{m} C$-adic completion of $C$ and $\phi: \hat{R} \rightarrow \hat{C}$ the canonical map on completions extending the inclusion $R \hookrightarrow C$. Let $\mathbf{a}=\operatorname{ker}(\phi)$. We have

$$
R \hookrightarrow \hat{R} / \mathbf{a} \hookrightarrow \hat{C}
$$

with $\hat{C}$ a finite $\hat{R} / \mathbf{a}$-module by, for example, [Ma1, Theorem 8.4, page 58], and the map $R \rightarrow \hat{R} / \mathbf{a}$ an injection since the composite map $R \rightarrow C \rightarrow \hat{C}$ is an injection. We note also that since $\hat{C}$ is faithfully flat over $C$, the nonzero elements of $R$ are regular elements of $\hat{R} / \mathbf{a} \subseteq \hat{C}$. Therefore every associated prime of $\mathbf{a}$ in $\hat{R}$ is in the generic formal fiber of $R$ and the nonzero elements of $R$ are mapped by $\phi$ to regular elements of $\hat{R} / \mathbf{a}$. Let $\phi^{\prime}$ denote the canonical map of $\hat{R}[C] \cong \hat{R} \otimes_{R} C$ to $\hat{C}$ that extends $\phi$ and the canonical injection of $C \rightarrow \hat{C}$, let $\mathbf{b}=\operatorname{ker}\left(\phi^{\prime}\right)$, and set $D=\hat{R}[C] / \mathbf{b}$. Then 


$$
\hat{R} / \mathbf{a} \subseteq D \subseteq \hat{C}
$$

with $\hat{C}$ a finite integral extension of $\hat{R} / \mathbf{a}$. Thus $D=\hat{R}[C] / \mathbf{b}$ is a complete semilocal Noetherian subring of $\hat{C}$ and $\hat{C}$ is a finitely generated $D$-module. Moreover, if $\mathbf{n}$ is the Jacobson radical of $C$, then $C / \mathbf{n} \cong D / \mathbf{n} D \cong \hat{C} / n \hat{C}$. Hence by Nakayama's lemma (cf. [Ma1, Theorem 2.2]), $D=\hat{C}$. Therefore any element of $\hat{C}=\hat{R}[C] / \mathbf{b}$ is multiplied by a nonzero element of $R$ into $\hat{R} / \mathbf{a}$. Since $\hat{C}$ is a finitely generated $\hat{R} / \mathbf{a}$-module, it follows that there exists $t \in R, t \neq 0$, such that $t \hat{C} \subseteq \hat{R} / \mathbf{a}$. Thus if we set $A=(\hat{R} / \mathbf{a}) \cap K$, then $t C=t \hat{C} \cap K \subseteq A$. We record the following conclusions from these observations.

THEOREM 2.3. With notation as in (2.2), we have

(i) There is a nonzero conductor of $A=(\hat{R} / \mathbf{a}) \cap K$ into $C$, so if $A$ is Noetherian, then $C$ is a finitely generated $A$-module.

(ii) If $\hat{C}$ is reduced, i.e., $C$ is analytically unramified in the $\mathbf{m} C$-adic topology, then associated to $C$ there is an ideal $\mathbf{a}$ of $\hat{R}$ that is a finite intersection of primes in the generic formal fiber of $R$ such that $\hat{C}$ is a finite integral extension of $\hat{R} / \mathbf{a}$ inside the total quotient ring of $\hat{R} / \mathbf{a}$.

(iii) If $C$ is analytically irreducible, then there is a prime ideal $\mathbf{p}$ of $\hat{R}$ in the generic formal fiber of $R$ such $\hat{C}$ is a finite birational extension of $\hat{R} / \mathbf{p}$. In particular, $C$ is the contraction to $K$ of a finite birational integral extension of $\hat{R} / \mathbf{p}$.

(iv) If $C$ is analytically unramified and normal, if $\mathbf{a}$ is the kernel of the canonical map of $\hat{R} \rightarrow \hat{C}$, and if $B$ is the integral closure of $\hat{R} / \mathbf{a}$ in its total quotient ring, then $C=B \cap K$.

Proof. The assertions in (i), (ii) and (iii) follow from the observations in (2.2), and to see (iv), it suffices to show that if $C$ is an analytically unramified normal semilocal Noetherian domain with quotient field $K$ and if $B$ is the integral closure of $\hat{C}$ in the total quotient ring of $\hat{C}$, then $C=B \cap K$. If $x \in K$ is integral over the the faithfully flat extension $\hat{C}$ of $C$, then as in [Na, proof of (37.4)], we have $x=a / b$, with $a, b \in C$ and $a^{n} \in\left(a^{n-1} b, \ldots, a b^{n-1}, b^{n}\right) \hat{C}$ for some positive integer $n$. Therefore $a^{n} \in\left(a^{n-1} b, \ldots, b^{n}\right) \hat{C} \cap C=\left(a^{n-1} b, \ldots, b^{n}\right) C$, and it follows that $x$ is integral over $C$. Since we are assuming that $C$ is normal, it follows that $x \in C$.

We have the following corollary of (1.27) and (2.3). 
COROLlary 2.4. Suppose $(R, \mathbf{m})$ is a local Noetherian domain of positive dimension. Then $\alpha(R)$, the dimension of the generic formal fiber of $R$, is $\operatorname{dim}(R)-1$ if and only if $R$ is birationally dominated by a Noetherian valuation domain $V$ such that $V / \mathbf{m} V$ is a finite $R$-module.

One of the interesting aspects of the normal local Noetherian birational extensions $C$ of $R$ gotten in Theorem 1.6 is that, by Theorem 1.12, if $B$ is an integral domain, then $\hat{C}$ is an integral domain, i.e., $C$ is analytically irreducible. This allows us to make the following one-to-one correspondence. (We remark that in general a normal local Noetherian domain need not be analytically irreducible [ $\mathrm{Na}$, Example 7, page 209].)

Theorem 2.5. Let $(R, \mathbf{m})$ be an $n$-dimensional universally catenary local Noetherian domain with quotient field $K$ and $\mathbf{m}$-adic completion $\hat{R}$. Let $\alpha$ denote the dimension of the generic formal fiber $\hat{R} \bigotimes_{R} K=\hat{R}[K]$ of $R$, and assume that for each prime $\mathbf{p} \subseteq \hat{R}$ with $\mathbf{p} \cap R=0$ and $\mathrm{ht}(\mathbf{p})=\alpha$ the condition of $(1.6)$ is satisfied, i.e., for all but at most finitely many of the primes $\mathbf{q} \supset \mathbf{p}$, the ring $R /(\mathbf{q} \cap R)$ is complete.

(i) There is a one-to-one correspondence between the primes $\mathbf{p}$ of height $\alpha$ in the generic formal fiber of $R$ and analytically irreducible normal local Noetherian domains $C$ such that $C$ birationally dominates $R, \operatorname{dim}(C)=n-\alpha$, and $C / \mathbf{m} C$ is a finite $R$-module.

(ii) There is a one-to-one correspondence between ideals $\mathbf{a}$ of $\hat{R}$ that are a finite intersection of prime ideals of height $\alpha$ in the generic formal fiber of $R$ and normal semilocal analytically unramified Noetherian domains $C$ that birationally dominate $R$ such that each maximal ideal of $C$ has height $\alpha$, and $C / \mathbf{m} C$ is a finite $R$-module.

Proof. We have already observed in (2.3) that to each such $C$ there is associated an ideal $\mathbf{a}$ of $\hat{R}$ that is a finite intersection of primes in the generic formal fiber of $R$ and that if $B$ is the integral closure of $\hat{R} / \mathbf{a}$, then $B \cap K=C$. With our assumption that $R$ is universally catenary it follows that for any prime $\mathbf{p}$ of $\hat{R}$, we have $h t(\mathbf{p})+\operatorname{dim}(\hat{R} / \mathbf{p})=n$ [Ma1, Theorem 31.7]. Thus to each such $C$ we have associated an ideal $\mathbf{a}$ that is a finite intersection of prime ideals of height $\alpha$ in the generic formal fiber of $R$; and if $C$ is also analytically irreducible, then the associated ideal $\mathbf{a}$ is a prime ideal.

Conversely, to each such a, Theorems 1.6 and 1.12 imply that if $B$ is the integral closure of $\hat{R} / \mathbf{a}$, then $B \cap K=C$ is an analytically unramified normal 
semilocal Noetherian domain such that each maximal ideal of $C$ contains $\mathbf{m}$ and is of height $\alpha$, and $C / \mathbf{m} C$ is a finite $R$-module.

(2.6) We remark that Theorem 2.5 applies to several of the examples discussed in Section 1. If $(R, \mathbf{m})$ is a geometric local domain as in Example 1.6A, then there is a one-to-one correspondence between prime ideals $\mathbf{p}$ of dimension 1 in the generic formal fiber of $R$ and rank-one discrete valuation domains $C$ such that $C$ birationally dominates $R$ and $C / \mathbf{m} C$ is a finitely generated $R$-module. We also have a one-to-one correspondence between finite intersections of prime ideals of dimension 1 in the generic formal fiber of $R$ and normal semilocal Noetherian domains $C$ of dimension 1 such that $C$ birationally dominates $R$ and $C / \mathbf{m} C$ is a finitely generated $R$-module. Since any normal semilocal Noetherian domain of dimension 1 is analytically unramified, it is not necessary to hypothesize this condition on the $C$ in making the above correspondence. For the examples $(R, \mathbf{m})$ with $\alpha(R)<\operatorname{dim}(R)-1$ discussed in (1.22)-(1.24), Theorem 2.5(i) implies the existence of a one-to-one correspondence between prime ideals $\mathbf{p}$ in the generic formal fiber of $R$ of height $\alpha(R)$ and normal analytically irreducible local Noetherian domains $C$ such that $C$ birationally dominates $R, C / \mathbf{m} C$ is a finitely generated $R$-module, and $\operatorname{dim}(C)=\operatorname{dim}(R)-\alpha(R)$. Similarly, Theorem 2.5(ii) implies the existence of a one-to-one correspondence between finite intersections of these primes and normal semilocal analytically unramified Noetherian domains $C$ that birationally dominate $R$ with each maximal ideal of $C$ of height $\alpha(R)$ and $C / \mathbf{m} C$ a finite $R$-module.

It is known [R3, Corollary 3.2] (cf. (1.24) above) that in general, prime ideals maximal with respect to being in the generic formal fiber need not have the same height. We note the following result that is similar to (2.5).

Theorem 2.7. Let $(R, \mathbf{m})$ be a local Noetherian domain with quotient field $K$ and $\mathbf{m}$-adic completion $\hat{R}$. Assume that for every prime ideal $\mathbf{p}$ of $\hat{R}$ that is maximal in the generic formal fiber of $R$ the conditions of (1.6) are satisfied, i.e., for all but at most finitely many of the primes $\mathbf{q} \supset \mathbf{p}$ of $\hat{R}, R /(\mathbf{q} \cap R)$ is complete.

(i) There is a one-to-one correspondence between the primes $\mathbf{p}$ of $\hat{R}$ that are maximal in the generic formal fiber of $R$ and analytically irreducible normal local Noetherian domains $C$ that birationally dominate $R$ and have $\alpha(C)=0$ and $C / \mathbf{m} C$ is a finite $R$-module.

(ii) There is a one-to-one correspondence between ideals a of $\hat{R}$ that are finite intersections of prime ideals maximal in the generic formal fiber of $R$ and 
analytically unramified normal semilocal domains $C$ which birationally dominate $R$, have $\alpha(C)=0$, and have $C / \mathbf{m} C$ is a finite $R$-module.

Proof. By Theorems 1.6 and 1.12, with each such $\mathbf{a}$ we get that if $B$ is the integral closure of $\hat{R} / \mathbf{a}$ in its total quotient ring, then $C=B \cap K$ is a normal semilocal Noetherian domain with $\mathbf{m} C$ contained in the Jacobson radical of $C$ and $C / \mathrm{m} C$ a finite $R$-module, and also $\hat{C} \subseteq B$, so $C$ is analytically unramified, and if $\mathbf{a}$ is prime, then $C$ is analytically irreducible. Moreover, the fact each minimal prime of $\mathbf{a}$ is maximal in the generic formal fiber of $R$ and $\hat{C} \subseteq B$ implies that $\alpha(C)=0$. Conversely, if $C$ has the stated conditions and $\mathbf{a}$ is the kernel of the canonical map $\hat{R} \rightarrow \hat{C}$, then by parts (ii)-(iv) of Theorem 2.3, $C=B \cap K$ where $B$ is the integral closure of $\hat{R} / \mathbf{a}$ in its total quotient ring. The fact that $\alpha(C)=0$ implies that each minimal prime of $\mathbf{a}$ is maximal in the generic formal fiber of $R$, and we have that $\mathbf{a}$ is prime if and only if $C$ is analytically irreducible.

(2.8) As we have remarked earlier, it seems difficult to determine the heights of all prime ideals maximal in the generic formal fiber of a local Noetherian domain. Using the fact that a complete local Noetherian ring of positive dimension is of uncountable cardinality, we note in (4.10) below some good properties of primes maximal in the generic formal fiber of a countable local Noetherian domain. But, for example, it seems to be unknown whether all prime ideals maximal in the generic formal fiber of an uncountable geometric local domain of dimension $n$ are of height $n-1$. We remark that Theorem 2.7 applies to the example $B$ of [R3, Corollary 3.2] discussed in (1.24) if and only if the two classes of prime ideals maximal in the generic formal fiber of $B$ indicated in (1.25) are indeed the only prime ideals maximal in the generic formal fiber of $B$.

(2.9) We remark that if $C$ as in (2.5) or (2.7) is either one-dimensional or excellent, then the number of maximal ideals of $C$ is the same as the number of minimal primes of $\hat{C}$ [Na, page 122 and (37.9)], [K, Corollary 5], and hence the same as the number of minimal primes of the ideal $\mathbf{a}$ of $\hat{R}$ corresponding to $C$. But the existence of normal local Noetherian domains of dimension 2 that are analytically unramified but not analytically irreducible [ $\mathrm{Na}$, Example 7, page 209] shows that the correspondence can give a local $C$ for which the associated ideal a has more than one minimal prime.

\section{On the excellence of birational extensions}

With notation as in the introduction, since it is usually the case that the bira- 
tional extension $C=(\hat{R} / \mathbf{a}) \cap K$ is not a spot over $R$ (cf. Theorem $1.6(\mathrm{v}))$, it is to be expected that $R$ may excellent and yet $C$ fail to be excellent. Indeed, it is often the case that there exist Noetherian $C$ birationally dominating $R$ which fail to be excellent.

Remark 3.1. If $\operatorname{dim}(R)>1$ and $\alpha(R)=\operatorname{dim}(R)-1$, then by Corollary 1.27, there exists a one-dimensional local Noetherian domain $C=(\hat{R} / \mathbf{a}) \cap K$ birationally dominating $R$ that is analytically ramified and therefore not excellent.

(3.2) As we mentioned in the introduction, the condition that the formal fibers of a Noetherian ring $A$ be geometrically regular is an important part of the definition that $A$ be excellent. If $(R, \mathbf{m})$ is a local Noetherian domain, if $\mathbf{a}$ is a finite intersection of prime ideals in the generic formal fiber of $R$ all of the same dimension, if $B$ is the integral closure of $\hat{R} / \mathbf{a}$ and $C=B \cap K$, and if for all but at most finitely many of the primes $\mathbf{q} \supseteq \mathbf{a}$ of $\hat{R}$, the ring $R /(\mathbf{q} \cap R)$ is complete, then by Theorem 1.12, $C$ is universally catenary. Therefore $C$ is excellent if and only if the formal fibers of $C$ are geometrically regular [G, (7.7.3), page 214].

In considering geometric regularity of the formal fibers, the field characteristic naturally plays an important role and we have some distinctions according to the characteristic of $R$. For example, we have

Proposition 3.3. Suppose $(R, \mathbf{m})$ is a local Noetherian domain with field of fractions $K$ and $\operatorname{dim}(R)=n>1$. Suppose $\mathbf{p} \in \operatorname{Spec}(\hat{R})$ is in the generic formal fiber of $R$ and $\operatorname{dim}(\hat{R} / \mathbf{p})=1$. Then $C=(\hat{R} / \mathbf{p}) \cap K$ is a one-dimensional local Noetherian domain. If $R$ is of characteristic zero, then $C$ is excellent. On the other hand, if $R$ is regular of characteristic $p>0$ and contains a perfect coefficient field $k$, then $C$ is not excellent.

Proof. By (1.27), $C$ is an analytically irreducible one-dimensional local Noetherian domain. Therefore $C$ is universally catenary [Ma1, Theorem 31.6, page 251], and the formal fibers of $C$ are regular, so if $\operatorname{char}(C)=0$, the formal fibers of $C$ are geometrically regular and $C$ is excellent [G, (7.83), page 214]. On the other hand, suppose $\operatorname{char}(R)=p>0$ and $R$ is regular with a perfect coefficient field $k$. To show that $C$ fails to be excellent, or even be a Nagata ring, it suffices to show that the field of fractions $L$ of $\hat{C}$ is not separable as an extension field of $K$, the field of fractions of $C$ [Ma3, Theorem 71, page 237]. The integral closure of $\hat{C}$ is a complete DVR of the form $k^{\prime}[[t]]$ where $k^{\prime}$ is a finite algebraic extension of $k$, so $L=k^{\prime}((t))$. Let $x_{1}, \ldots, x_{n}$ be a regular system of parameters for 
$R$. Then we have $\left[K\left(x_{1}^{1 / p}, \ldots, x_{n}^{1 / p}\right): K\right]=p^{n}>p$, while $x_{1}^{1 / p}, \ldots, x_{n}^{1 / p} \in$ $k^{\prime}\left[\left[t^{1 / p}\right]\right]$, so $\left[L\left(x_{1}^{1 / p}, \ldots, x_{n}^{1 / p}\right): L\right] \leq p$. Therefore $L / K$ is not linearly disjoint from $K^{1 / p} / K$, i.e., $L / K$ is not separable.

Example 3.4. Suppose $k$ is a perfect field, $x, y$ are indeterminates over $k$ and $R=k[x, y]_{(x, y)}$. If $\mathbf{p}$ is a nonzero prime ideal in the generic formal fiber of $R$, then the one-dimensional local Noetherian domain $C=(\hat{R} / \mathbf{p}) \cap k(x, y)$ is excellent if $\operatorname{char}(k)=0$, and is not excellent if $\operatorname{char}(k)=p>0$.

THEOREM 3.5. Suppose $(\boldsymbol{R}, \mathbf{m})$ is a local domain containing a field of characteristic 0 and $\mathbf{p}$ is a prime ideal in the generic formal fiber of $R$ such that $\hat{R} / \mathbf{p}$ is normal and each prime ideal $\mathbf{q}$ of $\hat{R}$ with $\mathbf{q}>\mathbf{p}$ is extended from $R$ and for all but at most finitely many of the $\mathbf{q}, R /(\mathbf{q} \cap R)$ is complete. If $R$ is excellent, then $C=(\hat{R} / \mathbf{p}) \cap K$ is excellent.

Proof. To show that $C$ is excellent, it suffices to show that $C$ is universally catenary and that the formal fibers of $C$ are geometrically regular. By Theorem $1.12,(\hat{R} / \mathbf{p})=\hat{C}$ is the completion of $C$ and $C$ is universally catenary. Let $\mathbf{u}$ be a nonzero prime ideal of $C$ and let $\mathbf{v}=\mathbf{u} \cap R$. Since $\hat{R} / \mathbf{p}=\hat{C}$ is faithfully flat over $C$, there exists a prime ideal $\mathbf{q} / \mathbf{p}$ of $\hat{R} / \mathbf{p}$ that lies over $\mathbf{u}$ in $C$. Therefore $\mathbf{q} \cap R=\mathbf{u} \cap R=\mathbf{v}$. By hypothesis, the prime ideal $\mathbf{q}$ of $\hat{R}$ is extended from $R$. Hence $\mathbf{v} \hat{R}=\mathbf{q}$, and $\mathbf{v} \hat{C}=(\mathbf{q} / \mathbf{p}) \hat{C}=\mathbf{u} \hat{C}$. If $V=R-\mathbf{v}$, then since $R$ is excellent, the formal fiber $V^{-1}(\hat{R} / \mathbf{q})$ is regular. We have $\hat{R} / \mathbf{q}=\hat{C} / \mathbf{u} \hat{C}$, and $V \subseteq U=$ $C-\mathbf{u}$, so $U^{-1}(\hat{C} / \mathbf{u})$ is a localization of $V^{-1}(\hat{R} / \mathbf{q})$ and hence is regular. Since $R$ and hence $C$ contains a field of characteristic zero, it follows that the formal fibers of $C$ are geometrically regular. Therefore $C$ is excellent.

We remark that examples to which Theorem 3.5 applies are described in (1.22) -(1.24) above. Another situation in which $C$ is excellent is the following.

ExAmPle 3.6. Let $k$ be a field of characteristic zero, let $x_{1}, \ldots, x_{d}, y, t$ be indeterminates over $k$, let $S$ be the $d$-dimensional regular local ring $S=k\left[x_{1}, \ldots, x_{d}\right]_{\left(x_{1}, \ldots, x_{d}\right)}$, and suppose $\tau=\sum_{i=1}^{\infty} b_{i} y^{i} \in k[[y]]$ is a formal power series algebraically independent over $S[y]$. Let $B$ be the $d+2$-dimensional regular local ring $R=S[y, t]_{\left(\mathbf{m}_{S}, t, t\right)}$. Then $\hat{R}$ is the formal power series ring $k\left[\left[x_{1}, \ldots\right.\right.$, $\left.\left.x_{d}, y, t\right]\right]$ and $\mathbf{p}=(t-\tau) \hat{R}$ is a height one prime in the generic formal fiber of $R$. Let $K$ denote the field of fractions of $R$. We wish to observe that $C=(\hat{R} / \mathbf{p})$ $\cap K$ is a $d+1$-dimensional excellent regular local domain. 
Proof. It is clear that $V=k(y, \tau) \cap k[[y]]$ is a rank-one discrete valuation domain with completion $k[[y]]$.

If for $n \in \mathbf{N}$ we define

$$
\tau_{n}=\sum_{i=n}^{\infty} b_{i} y^{i-n}
$$

then we have the relations

$$
\tau_{n}=b_{n}+\tau_{n+1} y
$$

Hence if we define

$$
V_{n}=k\left[y, \tau_{n}\right]_{\left(y, \tau_{n}-b_{n}\right)} \text { and } \quad C_{n}=k\left[x_{1}, \ldots, x_{d}, y, \tau_{n}-b_{n}\right]_{\left(x_{1}, \ldots, x_{d}, y, \tau_{n}-b_{n}\right)},
$$

then each $V_{n}$ is a 2 -dimensional regular local domain, each $C_{n}$ is a $d+2$-dimensional regular local domain, and we have

$$
V=\lim _{\rightarrow}\left(V_{n}\right) \text { and } V\left[x_{1}, \ldots, x_{d}\right]_{\left(y, x_{1}, \ldots, x_{d}\right)}=\lim _{\rightarrow}\left(C_{n}\right) .
$$

Moreover, $V\left[x_{1}, \ldots, x_{d}\right]_{\left(y, x_{1}, \ldots, x_{d}\right)}=A$ is a $d+1$-dimensional regular local domain that is a spot over the rank-one discrete valuation domain $V$. Since $V$ is of characteristic zero, $V$ is excellent, and hence $A$ is excellent.

We have $R \cong S[y, \tau]_{\left(\mathbf{m}_{S}, y, \tau\right)}$ as in the following diagram

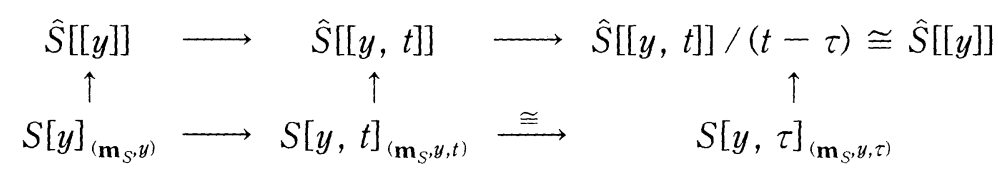

and with this identification, we have that $A$ is birationally dominated by $C=(\hat{R} / \mathbf{p}) \cap K$, and $\hat{A}=\hat{R} / \mathbf{p}$, so $A=C$. Therefore $C$ is a $d+1$-dimensional excellent regular domain local as we wished to show.

(3.7) In considering a situation where $S$, rather than being $k\left[x_{1}, \ldots, x_{d}\right]_{\left(x_{1}, \ldots, x_{d}\right)}$, is assumed to be a local Noetherian domain such that

$$
k\left[x_{1}, \ldots, x_{d}\right]_{\left(x_{1}, \ldots, x_{d}\right)} \subseteq S \subseteq k\left[\left[x_{1}, \ldots, x_{d}\right]\right]
$$

and such that $S$ has completion $k\left[\left[x_{1}, \ldots, x_{d}\right]\right]$, then more is required of $\tau \in k[[y]]$ in order to be able to realize $C=(\hat{R} / \mathbf{p}) \cap K$ as such a nice directed union. This situation is considered in [HR2]. 


\section{Non-Noetherian birational extensions associated with prime ideals in the generic formal fiber}

In this section we present two non-Noetherian examples of birational extensions associated with prime ideals in the generic formal fiber of a local Noetherian domain. The first example is due to Nagata and shows that the assumptions in Theorem 1.6 are not necessarily satisfied if the ring is not excellent (or at least not a Nagata ring). Therefore in a non-excellent local Noetherian domain $R$ there are possibly prime ideals $\mathbf{p}$ maximal in the generic formal fiber of $R$ such that for infinitely many prime ideals $\mathbf{q} \supseteq \mathbf{p}$, the ring $R /(\mathbf{q} \cap R)$ is not complete.

The second example is a modification of Ogoma's counterexample to the catenary chain conjecture. It actually provides two examples, the first shows that there is a height 2 prime ideal in the generic formal fiber of a localization of a polynomial ring in 6 variables over a field such that the associated birational extension to this prime is not Noetherian. This shows, in particular, that even in the case where $R$ is excellent, it is not possible to extend Theorem 2.5 to a one-to-one correspondence between all prime ideals in the generic formal fiber of $R$ and certain normal local Noetherian birational extensions of $R$. Ogoma's example also provides a prime ideal of height one in the generic formal fiber of a non-excellent Nagata ring whose associated birational extension is not Noetherian.

We would like to mention that in the case where $R$ is a localization of a polynomial ring over a field, we do not know whether there is a height one prime ideal in the generic formal fiber of $R$ for which the associated birational extension is not Noetherian. In this case, we have obtained only normal local Noetherian domains $C$ such that $\operatorname{dim}(C)=\operatorname{dim}(R)-1$, or birational spots over $R$ with $\operatorname{dim}(C)=\operatorname{dim}(R)$, including $R$ itself. These examples involve the concept of a power series remaining algebraically independent modulo a family of prime ideals. They are presented in [HR2].

We use $\mathscr{Q}(R)$ to denote the field of fractions of an integral domain $R$.

Nagata's Example. We consider the 3-dimensional regular local domain constructed by Nagata in [ $\mathrm{Na}$, (E3.1) and (E5.1), pages 206-208]. Let $k$ be a field with $\operatorname{char}(k)=2$ and $\left[k: k^{2}\right]=\infty$, and let $R$ be the subring of the formal power series ring $k[[x, y, z]], R=k^{2}[[x, y, z]][k]$. Nagata shows that $R$ is a 3-dimensional regular local domain with completion $\hat{R}=k[[x, y, z]], R<\hat{R}$, and $\hat{R}$ is integral over $R$. In particular, $\alpha(R)=0$.

Let $\left\{b_{m}, c_{n}\right\}_{m, n \in \mathbf{N}}$ be 2 -independent elements in $k$ and set 


$$
d=y\left(\sum_{i=1}^{\infty} b_{i} x^{i}\right)+z\left(\sum_{i=1}^{\infty} c_{i} x^{i}\right)
$$

Then Nagata shows that the integral closure $T$ of the local Noetherian domain $A=R[d]$ is not Noetherian.

We note that

Proposition 4.1. (i ) $\alpha(A)=0$.

(ii) The completion $\hat{A}$ of $A$ is a nonreduced ring with exactly one minimal prime $\mathbf{p}$.

(iii) $\hat{A} / \mathbf{p} \cong \hat{R}$, in particular, $\hat{A} / \mathbf{p}$ is regular.

(iv) $\mathscr{Q}(A) \cap(\hat{A} / \mathbf{p})=T$.

Proof. (ii) We have

$$
A=R[u] /\left(u^{2}-d^{2}\right) \subseteq \hat{R},
$$

where $u$ is another variable, and hence

$$
\hat{A}=\hat{R}[u] /\left(u^{2}-d^{2}\right)=\hat{R}[u] /(u-d)^{2} .
$$

This shows $\hat{A}$ is not reduced and has exactly one minimal prime $\mathbf{p}=(u-d) \hat{A}$.

(i) We have the following inclusions

$$
R \hookrightarrow A \hookrightarrow \hat{R} \hookrightarrow \hat{A}=\hat{R} \otimes_{R} A
$$

with the extensions $R \hookrightarrow A$ and hence $\hat{R} \hookrightarrow \hat{A}$ being finite. Since $\alpha(R)=0$, we also have $\alpha(A)=0$.

(iii) We clearly have $\hat{A} / \mathbf{p} \cong \hat{R}$.

(iv) The ring $\mathscr{Q}(A) \cap(\hat{A} / \mathbf{p})$ is a local Krull domain birationally dominating $A$ and hence $T$, and since $\hat{A} / \mathbf{p}$ is integral over $R$, we have $\mathscr{Q}(A) \cap(\hat{A} / \mathbf{p}) \subseteq T$, so $\mathscr{Q}(A) \cap(\hat{A} / \mathbf{p})=T$.

(4.2) This example of Nagata shows that the hypothesis in Theorem 1.6 about the prime ideals maximal in the generic formal fiber of the local Noetherian domain $R$ need not hold if $R$ is not a Nagata ring.

Ogoma's Example. We first recall Ogoma's construction in order to explain the modifications needed for our purposes. Let $k$ be a countable field of infinite but countable transcendence degree over its prime subfield. Let $x, y, z, w$ be variables over $k$ and let $S=k[x, y, z, w]_{(x, y, z, w)}$. As shown in [O1], there is a certain enumeration $\left\{p_{q}\right\}_{n \in \mathbf{N}}$ with $p_{1}=w$ of a set of representatives of the prime 
elements of $S$. We define

$$
q_{n}=\prod_{j=1}^{n} p_{j}
$$

and

$$
g=x+\sum_{j=1}^{\infty} a_{j} q_{j}^{j}, h=y+\sum_{j=1}^{\infty} b_{j} q_{j}^{j} \quad \text { and } \quad l=z+\sum_{j=1}^{\infty} c_{j} q_{j}^{j},
$$

where $a_{j}, b_{j}, c_{j}$ are elements in $k$ chosen to give special properties of the power series $g, h, l \in k[[x, y, z, w]]=\hat{S}$. The special choice of the enumeration $\left\{p_{n}\right\}_{n \in \mathbf{N}}$ not only gives that $g, h$ and $l$ are algebraically independent over $S$, but also yields that $g, h, l$ form part of a regular system of parameters for $\hat{S}$ and $(g, h, l) \hat{S}$ is a height 3 prime ideal in the generic formal fiber of $S[01$, page 158]. Ogoma's counterexample to the chain conjecture is the local Noetherian domain

$$
R=k(x, y, z, w) \cap(\hat{S} /(g h, g l) \hat{S}) .
$$

We use this example to show that if $u, v$ are additional variables, then a certain birational extension of the localized polynomial ring

$$
A=k[x, y, z, w, u, v]_{(x, y, z, w, u, v)}
$$

is not Noetherian.

Since the elements $g h, g l$ are algebraically independent over

$$
S=k[x, y, z, w]_{(x, y, z, w)},
$$

the prime ideal $(u-g h, v-g l) \hat{A}$ is in the generic formal fiber of $A$. (It is not maximal in the generic formal fiber as we show below.) Let

$$
C=k(x, y, z, w, u, v) \cap(\hat{A} /(u-g h, v-g l) \hat{A}) .
$$

We clearly have

$$
C \cong k(x, y, z, w, g h, g l) \cap k[[x, y, z, w]] .
$$

ClaIm 4.3. The maximal ideal $\mathbf{m}_{C}$ of $C$ is generated by $x, y, z, w$.

Proof. Let

$$
g_{n}=x+\sum_{j=1}^{n} a_{j} q_{j}^{j}, h_{n}=y+\sum_{j=1}^{n} b_{j} q_{j}^{j} \quad \text { and } \quad l_{n}=z+\sum_{j=1}^{n} c_{j} q_{j}^{j}
$$

Then by identifying $C$ with $k(x, y, z, w, g h, g l) \cap k[[x, y, z, w]]$ as in the following diagram 


$$
\begin{array}{cc}
\hat{A}=k[[x, y, z, w, u, v]] & \rightarrow \hat{A} /(u-g h, v-g l) \hat{A} \cong k[[x, y, z, w]] \\
\uparrow & \cong \quad \uparrow \\
A=k[x, y, z, w, u, v]_{(x, y, z, w, u, v)} & \cong \quad k[x, y, z, w, g h, g l]_{(x, y, z, w, g h, g l)}
\end{array}
$$

we see that

$$
\left(g h-g_{n} h_{n}\right) / q_{n}^{n} \text { and }\left(g l-g_{n} l_{n}\right) / q_{n}^{n} \in C \text {. }
$$

This shows that any polynomial in $(x, y, z, w, g h, g l) k[x, y, z, w, g h, g l]$ is in the ideal $(x, y, z, w) C$.

To show that the maximal ideal of $C$ is generated by $x, y, z$ and $w$, it is enough to show that

$$
C / w C \cong k[x, y, z]_{(x, y, z)} .
$$

Since $w C=w k[[x, y, z, w]] \cap C, w C$ is a prime ideal of $C$, and we have

$$
k[x, y, z]_{(x, y, z)} \subseteq C / w C \subseteq k[[x, y, z, w]] /(w) \cong k[[x, y, z]] .
$$

An element of $C$ has the form $\theta=F / G$, where $F, G \in k[x, y, z, w, g h, g l]$. If $G$ is a unit of $C$, or equivalently of $k[[x, y, z, w]]$, then it is clear that the image of $\theta$ in $C / w C$ is in $k[x, y, z]_{(x, y, z)}$, Suppose $G$ is not invertible in $k[[x, y, z, w]]$ and write $G=w^{e} G^{\prime} \in k[[x, y, z, w]]$, where $G^{\prime} \notin w k[[x, y, z, w]]$. Since $F / G \in C$, it follows that $F \in w^{e} C$. Pick $n \in \mathbf{N}$ with $n>e$ and write:

$$
g h=g_{n} h_{n}+q_{n}^{n} \sigma_{n} \quad \text { and } \quad g l=g_{n} l_{n}+q_{n}^{n} \tau_{n},
$$

where $\sigma_{n}, \tau_{n} \in C$. Regarding $F, G$ as polynomials in $k[x, y, z, w][g h, g l]$, we have

$$
F(g h, g l)=F\left(g_{n} h_{n}, g_{n} l_{n}\right)+q_{n}^{n} F_{1} \text { and } \quad G(g h, g l)=G\left(g_{n} h_{n}, g_{n} l_{n}\right)+q_{n}^{n} G_{1},
$$

where $F_{1}, G_{1} \in C$. Since $n>e$, it follow that

$$
F\left(g_{n} h_{n}, g_{n} l_{n}\right)=w^{e} f_{0} \text { and } G\left(g_{n} h_{n}, g_{n} l_{n}\right)=w^{e} g_{0},
$$

with $f_{0}, g_{0} \in S$. Therefore, we have

$$
\left.\theta=F / G=\left(f_{0}+\left(q_{n}^{n} / w^{e}\right) F_{1}\right) /\left(g_{0}+q_{n}^{n} / w^{e}\right) G_{1}\right) .
$$

Since $\left(q^{n} / w^{e}\right) \in w C$ and $g_{0} \notin w C$, the image of $\theta$ in $C / w C$ is the same as that of $f_{0} / g_{0}$. Therefore $C / w C \cong k[x, y, z]_{(x, y, z)}$, which completes the proof of Claim 4.3.

\section{Claim 4.4. $\quad C$ is not Noetherian.}


Proof. Suppose $C$ is Noetherian. Since the maximal ideal of $C$ is $(x, y, z$, w) $C$ and

$$
S=k[x, y, z, w]_{(x, y, z, w)} \subseteq C \subseteq k[[x, y, z, w]]=\hat{S},
$$

it follows [B1, Proposition 11, page 74] that $\hat{C}=k[[x, y, z, w]]$ and $C$ is a 4-dimensional regular local domain. Since $g h, g l \in C$, we see that each of the height one prime ideals $g \hat{C}, h \hat{C}, l \hat{C}$ contracts to $C$ to a principal height one prime of $C$, say $g \hat{C} \cap C=g_{1} C, h \hat{C} \cap C=h_{1} C$, and $l \hat{C} \cap C=l_{1} C$. Since $(g, h, l) \hat{C}$ is of height 3 , the prime ideals $g \hat{C}, h \hat{C}, l \hat{C}$ are distinct. We have $g h, g l \in g \hat{C} \cap$ $C=g_{1} C$, and $(h, l) \hat{C}$ is of height 2 , so $g \hat{C}=g_{1} \hat{C}$. It follows that $g_{1} C$ and $h_{1} C$ are distinct height one primes of $C$. We have $g h \in h_{1} C$ and $g h \hat{C}=g_{1} h \hat{C} \subseteq h_{1} \hat{C}$ $\subseteq h \hat{C}$. Since $h_{1} \notin g_{1} \hat{C}$, we conclude that $h_{1} \hat{C}=h \hat{C}$. In a similar way, it follows that $l_{1} \hat{C}=l \hat{C}$. Therefore the height 3 prime ideal $(g, h, l) \hat{S}=\left(g_{1}, h_{1}, l_{1}\right) \hat{S}$ is extended from $C$ and $\left(g_{1}, h_{1}, l_{1}\right) C$ is a prime ideal of $C$ height 3 . But this stands in contradiction to the fact that $C$ is of transcendence degree 2 over $S$ and $(g, h, l) \hat{S}$ $\cap S=(0)$. We conclude that $C$ is not Noetherian.

Remark 4.5. There are several questions one might ask about the nonNoetherian local Krull domain $C$. Since $\mathbf{m}_{C}=(x, y, z, w) C$, the $\mathbf{m}_{C}$-adic completion of $C$ is $\hat{C}=k[[x, y, z, w]]$, and since $C$ is not Noetherian, there exists a finitely generated ideal in $C$ that is not closed in the $\mathbf{m}_{C}$-adic topology [ $\mathrm{Na}, 31.8$ ), page 110]. Since $C=\mathscr{Q}(C) \cap \hat{C}$, principal ideals in $C$ are closed. But it is unclear to us whether $C$ is a UFD or whether there exists a nonunit principal ideal $a \hat{C}$ such that $a \hat{C} \cap C$ is of height greater than one.

Remark 4.6. The ring $C$ provides also an example of a birational extension of a 5-dimensional regular local domain $D$ associated with a height one prime ideal in the generic formal fiber of $D$.

Proof. To construct $D$ we consider the ring $B=k(x, y, z, w, g h) \cap$ $k[[x, y, z, w]]$. The ring $B$ is, after relabeling the variables, the ring considered in [R1]. It is shown in [R1] that $B$ is a 4 -dimensional regular local domain with completion $k[[x, y, z, w]]$. Moreover, $B$ is a Nagata ring that is not excellent. Let $t$ be another variable and let $D=B[t]_{\left(\mathbf{m}_{B}, t\right)}$. Then $D$ is a 5 -dimensional regular local domain. Since $g l$ is algebraically independent over $B$, the prime ideal $(t-g l) \hat{D}$ is in the generic formal fiber of $D$, and we have

$$
C \cong \mathscr{Q}(D) \cap(\hat{D} /(t-g l) \hat{D}) .
$$


Remark 4.7. The prime ideal $(u-g h, v-g l) \subseteq k[[x, y, z, w, u, v]]=\hat{A}$ is not maximal in the generic formal fiber of $A$. Indeed, since $A$ is countable and $\hat{A}$ is equidimensional, it follows from (4.10) below that each prime ideal maximal in the generic formal fiber of $A$ is of dimension one and height equal to $\operatorname{dim}(A)-1$ $=5$. It is relatively easy to see that $(u-g h, v-g l, g-h l) \hat{A}$ is a prime ideal in the generic formal fiber of $A$ that properly contains $(u-g h, v-g l) \hat{A}$.

Proof. Since $g, h, l$ are part of a regular system of parameters of $\hat{S}=$ $k[[x, y, z, w]]$ and $(g, h, l) \hat{S}=\mathbf{q}$ is a height 3 prime in the generic formal fiber of $S$, and since $A=S[u, v]_{\left(\mathbf{m}_{S}, u, v\right)}$ and $\hat{A}=\hat{S}[[u, v]]$, we have

$$
(\hat{S} /(g-h l) \hat{S})[[u, v]] \cong \hat{S}[[u, v]] /(g-h l) \hat{S}[[u, v]]=\hat{A} /(g-h l) \hat{A}=T
$$

is a 5-dimensional regular local domain containing $A$ and the image $\mathbf{q}^{\prime}$ in $T$ of $\mathbf{q} \hat{A}$ is a height 2 prime of $T$ with $\mathbf{q}^{\prime} \cap A=(0)$. If $h^{\prime}, l^{\prime}$ are the images of $h, l$ in $T$, then $\mathbf{q}^{\prime}=\left(h^{\prime}, l^{\prime}\right) T$. We have that $(0)<h^{\prime} T<\mathbf{q}^{\prime}$ is a chain of prime ideals of $T$, so $\left(h^{\prime}, l^{\prime}\right) A\left[h^{\prime}, l^{\prime}\right]=\mathbf{u}$ is a height 2 prime of $A\left[h^{\prime}, l^{\prime}\right]$ with $\mathbf{u} \cap A=(0)$. It follows that $\mathscr{Q}(A)\left[h^{\prime}, l^{\prime}\right]$ is 2 -dimensional and therefore a polynomial ring in 2 variables over $\mathscr{Q}(A)$, so $h^{\prime}, l^{\prime}$ are algebraically independent over $A$.

The image of $(u-g h, v-g l, g-h l) \hat{A}$ in $\hat{A} /(g-h l) \hat{A}=T$ is $(u-$ $\left.\left(h^{\prime} l^{\prime}\right) h^{\prime}, v-\left(h^{\prime} l^{\prime}\right) l^{\prime}\right) T$, and $h^{\prime}, l^{\prime}$ algebraically independent over $A$ implies that $\left(h^{\prime} l^{\prime}\right) h^{\prime},\left(h^{\prime} l^{\prime}\right) l^{\prime}$ are also algebraically independent over $A$. Therefore the prime ideal $\left(u-\left(h^{\prime} l^{\prime}\right) h^{\prime}, v-\left(h^{\prime} l^{\prime}\right) l^{\prime}\right) T$ is in the generic fiber over $A$. It follows that $(u-g h, v-g l, g-h l) \hat{A}$, is in the generic formal fiber of $A$.

Remark 4.8. With notation as in (4.6), it is interesting to observe that

$$
B \cong(\hat{S}[[u]] /(u-g h) \hat{S}[[u]] \cap \mathscr{Q}(S)(u)
$$

and $D=B[t]_{\left(\mathbf{m}_{B}, v\right)} \cong B[v]_{\left(\mathbf{m}_{B}, v\right)}$, where the last isomorphism is obtained by mapping $t$ to $v$, With this identification, $D$ is a 5 -dimensional regular local domain that birationally dominates $A=S[u, v]_{\left(\mathbf{m}_{S}, u, v\right)}$ and we have the following diagram.

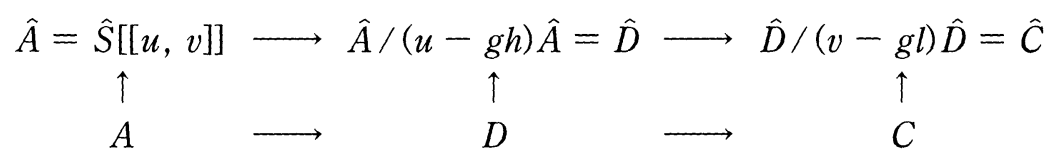

where the maps in the top row are surjections and in the bottom row are birational inclusions. We observed in (4.7) that $(u-g h, v-g l, g-h l) \hat{A}=\mathbf{w}$ is a height 3 prime ideal in the generic formal fiber of $A$. The image of $\mathbf{w}$ in $\hat{D}$ is a prime ideal in the generic formal fiber of $D$ that properly contains $(v-g l) \hat{D}$. Since $D$ is 
countable and the completion $\hat{D}$ of $D$ is equidimensional, it is true for $D$, as for $A$, that every prime ideal maximal in the generic formal fiber of $D$ is of dimension one and height equal to $\operatorname{dim}(D)-1=4$.

Lemma 4.9. Suppose $(\boldsymbol{R}, \mathbf{m})$ is a countable local Noetherian and $(S, \mathbf{n})$ is a complete local Noetherian ring that dominates $R$. If $\operatorname{dim}(S)>1$, then there exist uncountably many height one prime ideals $\mathbf{p}$ of $S$ such that $\mathbf{p} \cap R=(0)$. Moreover, all but countably many of the height one primes of $S$ are in the generic fiber over $R$.

Proof. Since $R$ is Noetherian and countable, $\operatorname{Spec}(R)$ is countable, and since $S$ is complete and $\operatorname{dim}(S)>1, \mathbf{n}$ properly contains any countable union of height one primes of $S$, cf [Bu, Lemma 3, page 371], so $S$ has uncountably many height one prime ideals. But each nonzero prime ideal of $R$ can be the contraction of at most finitely many height one primes of $S$. Therefore only countably many of the height one primes of $S$ have a nonzero contraction to $R$, and there exist uncountably many height one prime ideals $\mathbf{p}$ of $S$ such that $\mathbf{p} \cap R=(0)$.

Proposition 4.10. Suppose $(R, \mathbf{m})$ is a countable local Noetherian domain with $\operatorname{dim}(R)=n>0$, and let $\hat{R}$ denote the $\mathbf{m}$-adic completion of $R$. If $\mathbf{p} \in \operatorname{Spec}(\hat{R})$ is in the generic formal fiber of $R$ and if $\operatorname{dim}(\hat{R} / \mathbf{p})>1$, then there are uncountably many prime ideals of the generic formal fiber of $R$ that contain $\mathbf{p}$. Hence the generic formal fiber of $R$ is a Jacobson ring, and for each prime ideal $\mathbf{p}$ of $\hat{R}$ that is maximal in the generic formal fiber of $R$, we have $\operatorname{dim}(\hat{R} / \mathbf{p})=1$. If $\hat{R}$ is equidimensional we also have $\mathrm{ht}(\mathbf{p})=n-1$. Moreover, for each prime ideal $\mathbf{q} \in \operatorname{Spec}(\hat{R})$ with $\mathrm{ht}(\mathbf{q})>0$, there exists a prime ideal $\mathbf{p} \subset \mathbf{q}$ such that $\mathbf{p} \cap R=(0)$ and $\mathrm{ht}(\mathbf{q} / \mathbf{p})=1$.

Proof. If $\mathbf{p} \in \operatorname{Spec}(\hat{R})$ is in the generic formal fiber of $R$, then $S=\hat{R} / \mathbf{p}$ is a complete local domain that dominates $R$. If $\operatorname{dim}(S)>1$, then by (4.9) there exist uncountably many prime ideals of $S$ in the generic fiber over $R$, so $\mathbf{p}$ is contained in uncountably many prime ideals in the generic formal fiber of $R$. We conclude that the generic formal fiber of $R$ is a Jacobson ring, and $\operatorname{dim}(\hat{R} / \mathbf{p})=1$ for each prime ideal $\mathbf{p}$ maximal in the generic formal fiber of $R$. Since $\hat{R}$ is catenary, if $\hat{R}$ is equidimensional, then $\mathrm{ht}(\mathbf{p})=n-1$ for each such $\mathbf{p}$.

Suppose $\mathbf{q} \in \operatorname{Spec}(\hat{R})$ with $h t(\mathbf{q})>0$. If $h t(\mathbf{q})=1$, then for any minimal prime $\mathbf{p} \subset \mathbf{q}$ of $\hat{R}$, we have $\mathbf{p} \cap R=(0)$. On the other hand, if $h t(\mathbf{q})>1$, then as in (4.9), $\mathbf{q}$ properly contains the union of any countable set of height one prime ideals contained in $\mathbf{q}$, so there exist uncountably many height one primes of $\hat{R}$ contained in q. By (4.9), all but countably many of these height one prime ideals are 
in the generic formal fiber of $R$. Hence there exists a height one prime $\mathbf{u} \subset \mathbf{q}$ in $\hat{R}$ such that $\mathbf{u} \cap R=(0)$. Passing to $S=\hat{R} / \mathbf{u}$ and iterating the argument if necessary on $R \hookrightarrow S$, we conclude the existence of a prime ideal $\mathbf{p} \subset \mathbf{q}$ in $\hat{R}$ such that $\mathbf{p} \cap R=(0)$ and $h t(\mathbf{q} / \mathbf{p})=1$.

\section{REFERENCES}

[B1] N. Bourbaki, Algèbre Commutative, Ch. 3, Hermann, Paris, 1961.

[B1] - Algèbre Commutative, Ch. 7, Hermann, Paris, 1965.

[BR] M. Brodmann and C. Rothaus, Local rings with bad sets of formal prime divisors, J. Algebra, 75 (1982), pp. 386-394.

[Bu] Lindsay Burch, Codimension and analytic spread, Proc. Camb. Phil. Soc., 72 (1972), pp. 369-373.

[G] A. Grothendieck, Élements de Géometric algebrique $I V_{2}$, I.H.E.S., Publ. math., 24, 1965.

[HHS] W. Heinzer, C. Huneke, and J. D. Sally, A criterion for spots, J. Math. Kyoto Univ., 26 (1986), pp. 667-671.

[HR1] W. Heinzer and C. Rotthaus, Formal fibers and complete homomorphic images, Proc. Amer. Math. Soc., to appear.

[HR2] - On a certain class of algebraically independent elements, in preparation.

[HS] William Heinzer and Judith D. Sally, Extensions of valuations to the completion of a local domain, J. Pure Appl. Algebra, 71 (1991), pp. 175-185.

[H] R. C. Heitmann, A non-catenary, normal, local domain, Rocky Mountain J. Math., 12 (1982), pp. 145-148.

[K] Daniel Katz, On the number of minimal prime ideals in the completion of a local domain, Rocky Mountain J. Math., 16 (1986), pp. 575-578.

[Ma1] Hideyuki Matsumura, Commutative Ring Theory, Cambridge Univ. Press, Cambrige, 1986.

[Ma2] - On the dimension of formal fibres of a local ring in: Algebraic Geometry and Commutative Algebra in Honor of Masayoshi Nagata, Kinokuniya, Tokyo.

[Ma3] - Commutative algebra, second edition, Benjamin/Cummings, Reading, Massachusetts, 1980 .

[Na] Masayoshi Nagata, Local Rings, Interscience, New York,1962.

[Ni1] Jun-ichi Nishimura, Note on Krull domains, J. Math. Kyoto Univ., 15 (1975), pp. $397-400$.

[Ni2] - On ideal-adic completion of noetherian rings, J. Math. Kyoto Univ., 21 (1981), pp. 153-169.

[O1] T. Ogoma, Non-catenary pseudo-geometric normal rings, Japan. J. Math., 6 (1980), pp. 147-163.

[O2] Cohen-Macaulay factorial domain is not necessarily Gorenstein, Mem. Fac. Sci. Kochi Univ., 3 (1982), pp. 65-74.

[03] - Existence of dualizing complexes, J. Math. Kyoto Univ., 24 (1984), pp. $27-48$.

[R1] Christel Rotthaus, Universell Japanische Ringe mit nicht offenen regulärem Ort, Nagoya Math. J., 74 (1979), pp. 123-135.

[R2] — Komplettierung semilokaler quasiausgezeichneter Ringe, Nagoya Math. J., 
76 (1979), pp. 173-180.

[R3] - On rings with low dimensional formal fibres, J. Pure Aure Algebra, 71 (1991), pp. 287-296.

[V] Paolo Valabrega, On two-dimensional regular local rings and a lifting problem, Annali della Scuola Normale Superiore di Pisa, 27 (1973), pp. 1-21.

[W] D. Weston, On descent in dimension two and non-split Gorenstein modules, J. Algebra, 118 (1988), pp. 263-275.

W. Heinzer

Department of Mathematics

Purdue University

West Lafayette, IN 47907, USA

C. Rotthaus

Department of Mathematics

Michigan State University

East Lansing, MI 48824, USA

J. D. Sally

Department of Mathematics

Northwestern University

Evanston, IL 60201, USA 